\title{
MODELLING OF STRESS-CORROSION CRACKING BY USING PERIDYNAMICS
}

\author{
Dennj De Meo ${ }^{1}$, Cagan Diyaroglu ${ }^{1}$, Ning Zhu ${ }^{1}$, Erkan Oterkus ${ }^{1}{ }^{*}$, M. Amir Siddiq ${ }^{2}$ \\ ${ }^{1}$ Department of Naval Architecture, Ocean and Marine Engineering, University of Strathclyde, Glasgow, \\ Lanarkshire, G4 0LZ, UK \\ ${ }^{2}$ School of Engineering, University of Aberdeen, Aberdeen, AB24 3UE, UK
}

\begin{abstract}
We present for the first time a numerical multiphysics peridynamic framework for the modelling of adsorbedhydrogen stress-corrosion cracking (SCC), based on the adsorption-induced decohesion mechanism. The material is modelled at the microscopic scale using microstructural data. First-principle studies available in the literature are used for characterizing the process of intergranular material strength degradation. The model consists of a polycrystalline AISI 4340 high-strength low-alloy (HSLA) thin, pre-cracked steel plate subjected to a constant displacement controlled loading and exposed to an aqueous solution. Different values of stress intensity factor (SIF) are considered, and the resulting crack propagation speed and branching behaviour are found to be in good agreement with experimental results available in the literature.
\end{abstract}

Keywords: stress-corrosion cracking; polycrystalline materials; peridynamics; grain boundary diffusion; crack branching; hydrogen adsorption-induced decohesion

\section{Introduction}

SCC can be defined as the progressive fracturing of the material due to the presence of noncyclic tensile stress and the exposure to some gaseous and/or liquid environments. SCC can develop in brittle and non-brittle materials, metals and non-metals and can have different morphologies such as intergranular SCC (I-SCC) and transgranular SCC (T-SCC). Crack branching is common in SCC-damaged materials and, in certain cases, the tensile stress necessary to trigger the phenomenon can be as low as 5\% of the yield stress (Lynch, 2011). Due to the substantial number of SCC critical environment-material combinations, a wide range of systems related to different industries are affected by this phenomenon such as pipelines, nuclear power systems, aerospace vehicles, boilers, cooling water systems and oil and gas drilling and production systems (Raja and Shoji, 2011).

The first catastrophic failures due to SCC occurred in the first years of the $19^{\text {th }}$ century: boiler explosions cost the loss of a large number of human lives. The phenomenon was first recognized in 1873 by W. H. Johnson in a laboratory experiment (Lynch, 2011b). Despite

* Corresponding author. Tel.: +44 (0)141 5483876

E-mail address: erkan.oterkus@strath.ac.uk (E. Oterkus) 
the resources and efforts dedicated to the study of this problem, catastrophic failures due to SCC still occur. Moreover, hydrogen is deemed one of the potential candidates to replace fossil fuels. SCC remains, thus, the object of continuous study by academic, industry and other institutions, aimed at, for instance, SCC prediction, SCC monitoring, designing of SCC environmental control procedures and designing of better SCC-resistant materials.

As comprehensively described in (Lynch, 2011b), various theoretical models are available in the literature for explaining the mechanisms that lead or just contribute to SCC. However, there is not much agreement about which mechanisms are more appropriate for particular material-environment combinations. The published literature abounds with SCC experimental data such as those reported in (Han et al., 1998), (Thomas and Bruemmer, 2000), (Oriani and Josephic, 1974), (Bechtle et al., 2009), (Mine and Kimoto, 2011), (Hirose and Mura, 1984), ( Hirose and Mura, 1984) and (Yagodzinskyy et al., 2010), but their usefulness is limited by the large amount of parameters contributing to the phenomenon. Moreover, the number of SCC numerical models is increasing in the scientific literature, and many of them are based on the coupling of fracture mechanics and classical continuum mechanics (CCM) theory within the finite element method (FEM) via implementation of the cohesive zone model $(\mathrm{CZM})$ by using cell mesh cohesive elements. Some studies of this kind are described in (Raykar et al., 2011), (Scheider et al., 2008), (Falkenberg et al., 2009), (Rimoli and Ortiz, 2010) and (Ahn et al., 2007), where hydrogen assisted SCC in high-strength steels is modelled. A similar approach is used in (Traidia et al., 2012), (Alvaro et al., 2014a) and (Alvaro et al., 2014b) for the modelling of hydrogen embrittlement in steel pipelines. Studies based on atomistic simulations, such as (Arnoux, 2010) and (Li et al., 2002), are also available. Despite the worthy prediction capabilities and the resulting important insights provided by the use of these numerical techniques, there are various limiting factors. First of all, due to the lack of enough computational power, the current timescales and length scales that can be considered in atomistic simulations are far from those needed in practical engineering applications. Secondly, FEM cell mesh cohesive elements do not allow the analysis of arbitrary crack paths and often lead to mesh dependence and numerical convergence issues. Furthermore, if adaptive remeshing procedures are adopted, numerical errors can arise and computational cost will increase. As mentioned in (Rabczuk and Belytschko, 2004), another issue is related to the possible overestimation of the fracture energy in the case of crack path not coincident with the element boundaries. Therefore, the past decades have seen the introduction, development, further refinement and application of more advanced numerical techniques suitable for the reproduction of complex fracture 
behaviours observed in experimental tests. Among these approaches, the extended finite element method (XFEM) is probably the most popular one (Gravouil et al., 2002) (Moës et al., 2002) (Moës et al. 1999). However, due to factors such as the complexity of the method, the difficulty in handling highly distorted meshes (e.g. problems with large deformations and fragmentation) and innacurate prediction of stress intensity factors, meshless methods are sometimes preferred with respect to XFEM, despite the generally higher computational cost (Rabczuk et al., 2010). In this regard, cracking particles method (CPM) that does not require an explicit crack representation has been successfully applied to solve static, dynamic, impact and explosive problems both in 2-D and 3-D domains (Rabczuk and Belytschko, 2004) (Rabczuk and Belytschko, 2007). Another meshless method worth of note is the extended element free Galerkin method (XEFGM) which can achieve satisfactory results with a significant reduction in computational time with respect to the CPM (Rabczuk and Zi, 2007). Finally, CCM does not have a length scale parameter and, thus, it is not readily usable for multi-scale analyses. Fracture mechanics crack growth criteria are not always available, especially in the case of new and complex engineering materials. Complex behaviours, such as crack nucleation, crack branching, coalescence of multiple cracks and crack arrest, are unlikely to be fairly predicted due to accuracy and numerical convergence issues (Madenci and Oterkus, 2014). In this study, a new continuum mechanics formulation with a length scale parameter that is suitable for failure prediction, peridynamics (PD), is utilized for modelling stress-corrosion cracking.

PD is a recently introduced generalization of CCM theory (Silling and Askari, 2005) and is deemed a potentially successful candidate for the numerical modeling of SCC damage due to the possibility to overcome some of the limitations of the numerical approaches mentioned earlier. In fact, PD presents several advantages. First of all, its mathematical formulation does not break down in the presence of discontinuities such as cracks. Various studies, such as (Madenci and Oterkus, 2014), (Oterkus et al., 2012) and (Silling and Askari, 2005), have revealed the capability of peridynamics in capturing complex phenomena such as crack nucleation, crack branching, coalition of multiple cracks and crack arrest in isotropic and non-isotropic materials. Moreover, there is no need for external crack growth criteria, rules to discern between crack nucleation and branching, or external equations for treating interfaces. On top of that, peridynamics is also suitable for investigating multi-scale problems (Askari et al., 2008) and multiphysics problems (Oterkus et al., 2014), (Gerstle et al., 2008).

Due to their better strength-to-weight ratio as compared to carbon steels, and despite their sensibility to SCC in certain environments such as moist air and water, HSLA steel is broadly 
used in different industries and systems. As argued by Lynch in (Lynch, 2011b), hydrogenbased SCC mechanisms are widely recognized in high-strength steel, often leading to intergranular brittle fracture where little plasticity can be observed, supporting the idea of a decohesion type of cracking caused by the local hydrogen weakening of interatomic bonds. Moreover, in the case of non-charged hydrogen material exposed to a hydrogen-bearing environment, the predominant SCC mechanism is expected to be hydrogen adsorptioninduced decohesion (AIDEC) (Lynch, 2011b).

In this study, the peridynamic framework is used to predict the fracture behaviour of a polycrystalline thin pre-cracked HSLA steel plate with random texture exposed to an aqueous solution, by using data retrieved from first-principle calculations for characterizing the process of intergranular material strength degradation. The material mechanical response is described by means of microstructural data, and displacement-controlled loads of different magnitude are considered. The paper provides comparisons between the obtained numerical crack propagation speeds and experimental data available in the literature. SCC has never been modelled before by using peridynamics. Therefore, the novelty of this paper is threefold: (1) the application of PD for the modelling of SCC, (2) the introduction of a novel peridynamic hydrogen grain boundary diffusion model and (3) the prediction of detailed and realistic geometrical features concerning SCC damage in a polycrystalline material (i.e., stable crack propagation, micro-branching and the resulting penetration of the corrosive solution within the structure) that facilitates hydrogen diffusion and accelerates the degradation of the material, which is a salient aspect in real cases. 


\begin{tabular}{|c|c|}
\hline \multicolumn{2}{|c|}{ Nomenclature } \\
\hline $\mathrm{SCC}$ & Stress-corrosion cracking \\
\hline AIDEC & Adsorption-induced decohesion \\
\hline SIF & Stress intensity factor \\
\hline HSLA & High-strength low-alloy \\
\hline CZM & Cohesive zone model \\
\hline FEM & Finite element method \\
\hline $\mathrm{CCM}$ & Classical continuum mechanics \\
\hline PD & Peridynamics \\
\hline $\mathrm{BCC}$ & Body-centered cubic \\
\hline DFT & Density functional theory \\
\hline UBER & Universal binding energy relation \\
\hline$H_{x}$ & Horizon of a generic particle $\mathbf{x}$ \\
\hline$\delta$ & Radius of the horizon $[\mathrm{m}]$ \\
\hline f & Mechanical response function $\left[\mathrm{N} / \mathrm{m}^{6}\right]$ \\
\hline$f_{d}$ & Hydrogen concentration response function $\left[\mathrm{mol} / \mathrm{m}^{6} \mathrm{~s}\right]$ \\
\hline$C(\mathbf{x}, t)$ & Hydrogen concentration field $\left[\mathrm{mol} / \mathrm{m}^{3}\right]$ \\
\hline$\dot{C}(\mathbf{x}, t)$ & Time derivative of hydrogen concentration field $\left[\mathrm{mol} / \mathrm{m}^{3} \mathrm{~s}\right]$ \\
\hline$c$ & Mechanical bond constant $\left[\mathrm{N} / \mathrm{m}^{6}\right]$ \\
\hline$d$ & Hydrogen bond constant $\left[\mathrm{m}^{-2} \mathrm{~s}^{-1}\right]$ \\
\hline$s$ & Bond stretch \\
\hline$s_{0}$ & Critical stretch \\
\hline $\mathbf{x}$ & Vector defining the position of a generic particle $\mathbf{x}$ \\
\hline $\mathbf{x}^{\prime}$ & Vector defining the position of a generic neighbour of particle $\mathbf{x}$ \\
\hline $\mathbf{y}$ & Vector defining the position of particle $\mathbf{x}$ in the deformed configuration \\
\hline $\mathbf{y}^{\prime}$ & Vector defining the position of particle $\mathbf{x}^{\prime}$ in the deformed configuration \\
\hline $\mathbf{b}(\mathbf{x}, t)$ & Body force density field $\left[\mathrm{N} / \mathrm{m}^{3}\right]$ \\
\hline$K_{I C}$ & Fracture toughness $[\mathrm{MPa} \sqrt{\mathrm{m}}]$ \\
\hline
\end{tabular}




\begin{tabular}{|c|c|}
\hline$h$ & Plate's thickness $[\mathrm{m}]$ \\
\hline$L$ & Plate's length $[\mathrm{m}]$ \\
\hline$W$ & Plate's width $[\mathrm{m}]$ \\
\hline E & Young's modulus $\left[\mathrm{N} / \mathrm{m}^{2}\right]$ \\
\hline$G_{c}$ & Critical energy release rate $[\mathrm{N} / \mathrm{m}]$ \\
\hline$u$ & Enforced displacement applied to the plate $[\mathrm{m}]$ \\
\hline$C_{i j}$ & Elastic moduli of the local stiffness matrix $\left[\mathrm{N} / \mathrm{m}^{2}\right]$ \\
\hline$[\mathrm{C}]$ & Local stiffness matrix \\
\hline $\mathrm{SHC}$ & Surface hydrogen concentration $\left[\mathrm{mol} / \mathrm{m}^{2}\right]$ \\
\hline SHCSV & Surface hydrogen concentration saturation value $\left[\mathrm{mol} / \mathrm{m}^{2}\right]$ \\
\hline$\Delta x$ & Grid spacing $[\mathrm{m}]$ \\
\hline$d t$ & Time step size $[\mathrm{s}]$ \\
\hline$c_{T 1}$ & Bond constant type $-1\left[\mathrm{~N} / \mathrm{m}^{6}\right]$ \\
\hline$c_{T 2}$ & Bond constant type- $2\left[\mathrm{~N} / \mathrm{m}^{6}\right]$ \\
\hline$\xi_{i j}$ & Undeformed bond length between particles $i$ and $j[\mathrm{~m}]$ \\
\hline$\theta$ & Bond angle with respect to the crystal orientation angle [rad] \\
\hline$\gamma$ & Crystal orientation angle [rad] \\
\hline$V_{j}$ & Volume of a generic neighbouring particle $j\left[\mathrm{~m}^{3}\right]$ \\
\hline$q_{A}$ & Number of peridynamic bonds along $A$ directions \\
\hline$q_{B}$ & Number of peridynamic bonds along $B$ directions \\
\hline$D_{g b}$ & Grain boundary diffusion coefficient $\left[\mathrm{m}^{2} / \mathrm{s}\right]$ \\
\hline$\psi$ & Hydrogen grain boundary coverage \\
\hline $\mathbf{u}(\mathbf{x}, t)$ & Displacement field at $\mathbf{x}[\mathrm{m}]$ \\
\hline $\mathbf{u}\left(\mathbf{x}^{\prime}, t\right)$ & Displacement field at $\mathbf{x}^{\prime}[\mathrm{m}]$ \\
\hline$\rho(\mathbf{x})$ & Mass density at $\mathbf{x}\left[\mathrm{Kg} / \mathrm{m}^{3}\right]$ \\
\hline$\ddot{\mathbf{u}}(\mathbf{x}, t)$ & Acceleration vector field $\left[\mathrm{m} / \mathrm{s}^{2}\right]$ \\
\hline$d V_{x^{\prime}}$ & Volume of a generic neighbouring particle $\mathbf{x}^{\prime}\left[\mathrm{m}^{3}\right]$ \\
\hline
\end{tabular}




\section{Peridynamic Theory}

In order to determine the response of materials and structures subjected to different loading conditions, CCM was introduced and has been used to analyse numerous engineering problems. CCM is based on a continuum approximation where the body (structure) comprises an infinite number of infinitesimal volumes called material points as shown in Fig. 1. According to $\mathrm{CCM}$, a material point can only interact with material points in its nearest neighbourhood (direct contact). Although this assumption works well for many problems of interest, the CCM equations are not valid if there is any discontinuity, such as a crack, within the structure. This is mainly due to the fact that the CCM's governing equations are partial differential equations, whose spatial derivatives are not defined along discontinuities.

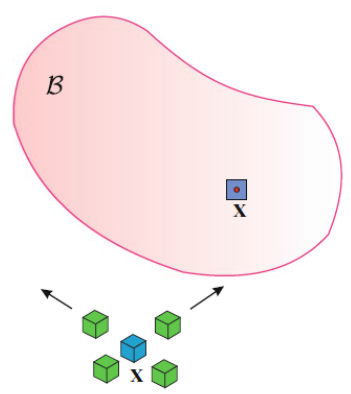

Fig. 1 - Classical continuum mechanics interactions.

In order to overcome this problem, a new continuum mechanics formulation, peridynamics, was introduced by Silling (Silling, 2000). According to this new formulation, a material point can interact with other material points not only within its nearest neighbourhood (direct contact), but also with material points comprised in a larger neighbourhood. Since the strength of the interaction between material points decreases as the distance between them increases, an influence domain, named horizon, $H_{\mathrm{x}}$, can be defined for each material point as shown in Fig. 2. Therefore, the material point $\mathbf{x}$ can only interact with material points within this domain. The radius of the horizon, $\delta$, is chosen depending on the nature of the problem in such a way that the model is able to fairly represent the physical mechanisms of interest (cf. (Seleson and Parks, 2011)). 


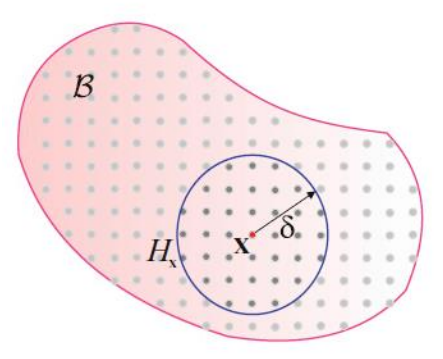

Fig. 2 - Peridynamics interactions.

The peridynamics' governing equation can be written as:

$$
\rho(\mathbf{x}) \ddot{\mathbf{u}}(\mathbf{x}, t)=\int_{H_{\mathbf{x}}} \mathbf{f}\left(\mathbf{u}\left(\mathbf{x}^{\prime}, t\right)-\mathbf{u}(\mathbf{x}, t), \mathbf{x}^{\prime}-\mathbf{x}\right) \mathrm{dV}_{\mathbf{x}^{\prime}}+\mathbf{b}(\mathbf{x}, t)
$$

where $\rho(\mathbf{x}), \ddot{\mathbf{u}}(\mathbf{x}, t)$ and $\mathbf{u}(\mathbf{x}, t)$ denote the density, acceleration and displacement of the material point $\mathbf{x}$ at time $t$, respectively. In Eq. (1), $\mathbf{f}\left(\mathbf{u}\left(\mathbf{x}^{\prime}, t\right)-\mathbf{u}(\mathbf{x}, t), \mathbf{x}^{\prime}-\mathbf{x}\right)$ represents the peridynamic force between material points $\mathbf{x}$ and $\mathbf{x}^{\prime}$ (also called mechanical response function). The term $\mathbf{b}(\mathbf{x}, t)$ is the body force acting on material point $\mathbf{x}$ at time $t$. Note that Eq. (1) is an integro-differential equation (i.e., time derivation and spatial integration) and is always valid regardless of any discontinuity in the structure.

In the case of an elastic material, the peridynamic force, as a result of the interaction (bond) between material points $\mathbf{x}$ and $\mathbf{x}^{\prime}$, can be expressed as:

$$
\mathbf{f}=\operatorname{css} \frac{\mathbf{y}^{\prime}-\mathbf{y}}{\left|\mathbf{y}^{\prime}-\mathbf{y}\right|}
$$

where $\mathbf{y}$ represents the location of the material point $\mathbf{x}$ in the deformed configuration, i.e., $\mathbf{y}=\mathbf{x}+\mathbf{u}$. In Eq. (2), the bond constant $c$ is the peridynamics' material parameter and the stretch parameter $s$ is defined as:

$$
s=\frac{\left|\mathbf{y}^{\prime}-\mathbf{y}\right|-\left|\mathbf{x}^{\prime}-\mathbf{x}\right|}{\left|\mathbf{x}^{\prime}-\mathbf{x}\right|}
$$

In the case of brittle material behaviour, the peridynamic force and the stretch relationship are shown in Fig. 3. 


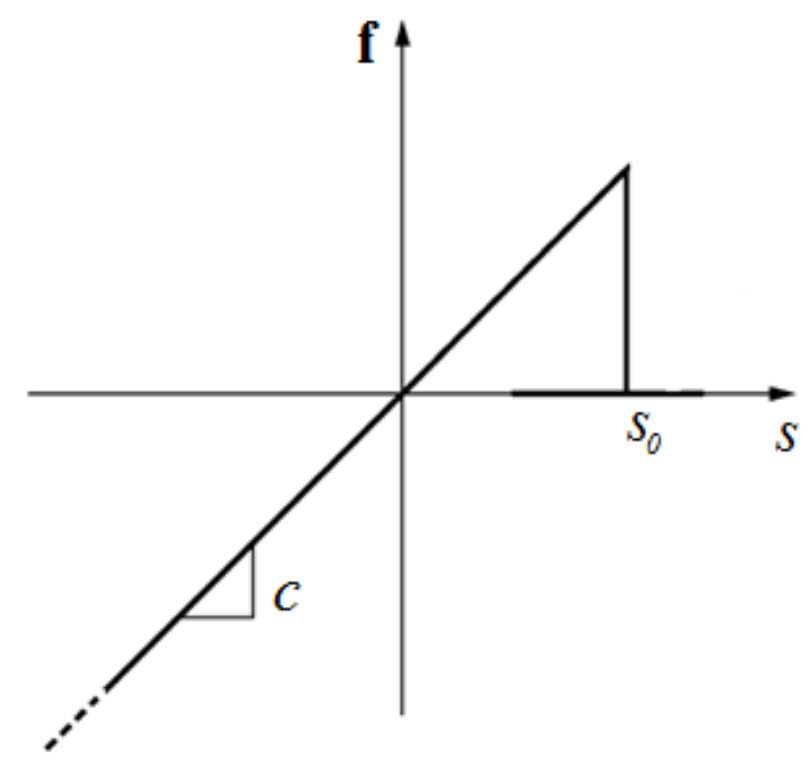

Fig. 3 - Definition of bond constant and critical stretch for linear elastic brittle material.

The parameter $s_{0}$ in Fig. 3 is called critical stretch. When the stretch of a generic peridynamic bond exceeds this critical value, the bond between the related two material points is broken. As a result, the peridynamic force between the two material points reduces to zero. With respect to the generally sophisticated failure criteria defined for different materials, the peridynamic definition of failure is pretty simple. Furthermore, there is no need to have an initial defect in the structure. Therefore, both crack initiation and propagation can be easily determined.

\section{Current Approach}

In this study, a microscopic material model is used to represent the behaviour of a polycrystalline structure with random texture. For this purpose, a new peridynamic material model (Section 3.1) is developed to express the deformation response of each crystal (Fig. 4). 


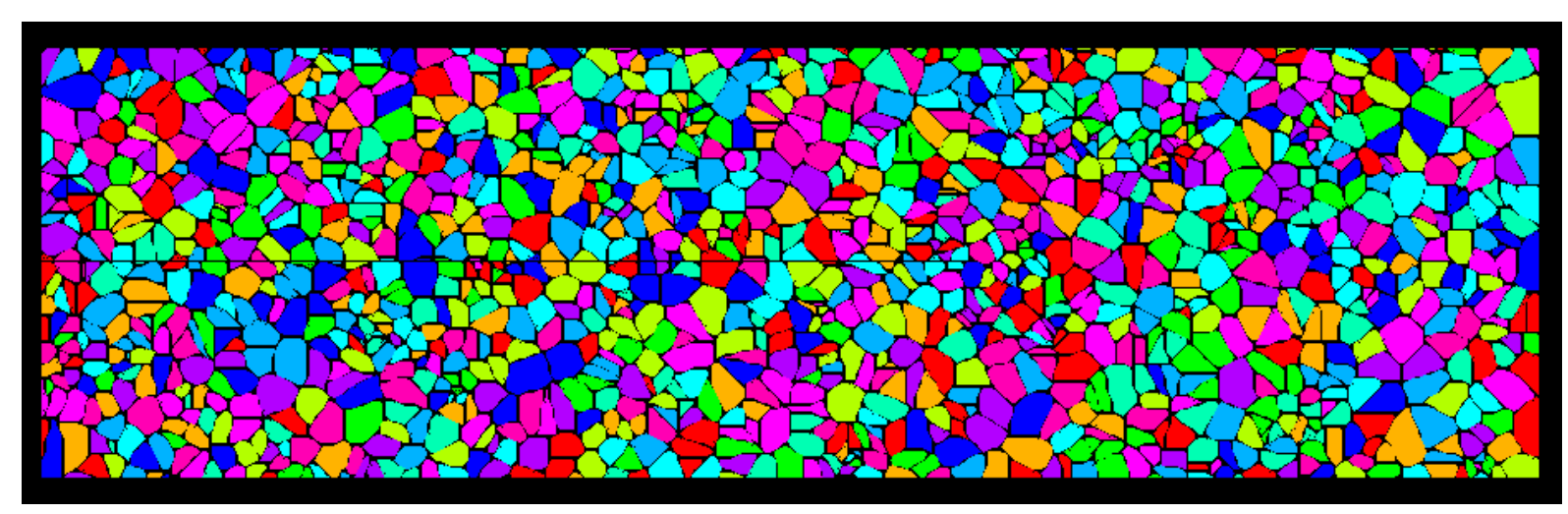

Fig. 4 - Polycrystalline material model.

Similar to the approach adopted in (Rimoli and Ortiz, 2010), (Serebrinsky et al. 2004), (Sofronis and McMeeking, 1989), (Scheider et al. 2008), (Alvaro et al. 2014a), (Olden et al. 2014) and (Raykar et al. 2011), hydrogen adsorption is treated by the application of appropriate boundary conditions. Fracture initiation due to the SCC hydrogen AIDEC mechanism is described by using the Fisher grain boundary diffusion model (Fisher, 1951). The resulting intergranular material embrittlement is modelled following an approach similar to that introduced in (Serebrinsky et al., 2004) and pursued in (Rimoli and Ortiz, 2010).

As mentioned earlier, peridynamics, originally introduced for the prediction of mechanical deformations and material failure, has been extended to represent other fields including thermal (Madenci and Oterkus, 2014), electrical (Gerstle et al. 2008) and moisture (Oterkus et al. 2014) fields. In the present study, a new hydrogen diffusion peridynamic formulation, based on the Fisher grain boundary diffusion model, is introduced.

Details about the new microscopic peridynamic model (Section 3.1), the novel peridynamic grain boundary diffusion formulation (Section 3.2), the hydrogen coverage boundary condition (Section 3.3) and the modelling of the resulting intergranular material strength degradation (Section 3.4) are provided in the next sections.

\subsection{Peridynamic micro-mechanical model}

The material considered in this study is HSLA steel. The fracture behaviour can be approximated by a linear elastic model (Anderson, 2005) and the microstructure is considered to be pure body-centered cubic (BCC). As shown in Fig. 5, two different peridynamic bonds are defined to represent the material behaviour:

- Type-1 bonds (dashed green lines) - exists in all directions (i.e., $\theta=0-2 \pi$ )

- Type-2 bonds (solid red lines) - exists only for the following directions: 


$$
\theta=\frac{\pi}{4}, \frac{3}{4} \pi, \frac{5}{4} \pi, \frac{7}{4} \pi
$$

Note that the angle $\theta$ is defined with respect to the orientation of the crystal. In the special case shown in Fig. 5, the crystal orientation $\gamma$ equals $\pi / 4$ and it is always measured with respect to the $\mathrm{x}$-axis. In general, an algorithm is used to assign a random orientation $\gamma$ to the grain. As a result of this procedure, when a polycrystalline system of random texture is represented by this model, type-2 bonds will exist in many different directions according to the random orientations of the crystals.

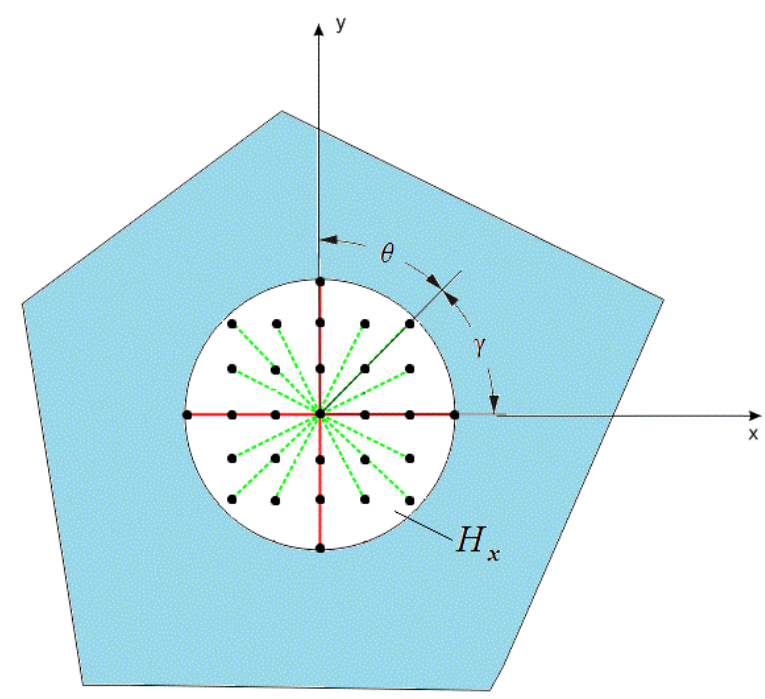

Fig. 5 - Type-1 (dashed green lines) and type-2 (solid red lines) bonds for the peridynamic micro-mechanical model.

The bond constants for type- 1 and type- 2 peridynamic bonds can be expressed in terms of the material constants of a cubic crystal, $C_{i j}$, by following a procedure similar to that explained in (Oterkus and Madenci, 2012). In the case of plane stress condition, the bond constants can be expressed as:

$$
c_{T 1}=\frac{12\left(C_{11}^{2}-C_{11} C_{12}\right)}{\pi h \delta^{3} C_{11}} \quad, \quad c_{T 2}=\frac{4\left(3 C_{11} C_{12}-2 C_{12}^{2}-C_{11}^{2}\right)}{\left(\beta_{A}+\beta_{B}\right) C_{11}}
$$

where $h$ is the thickness of the structure. The quantities $\beta_{A}$ and $\beta_{B}$ can be expressed as

$$
\beta_{A}=\sum_{j=1}^{q_{A}} \xi_{i j} V_{j} \quad, \quad \beta_{B}=\sum_{j=1}^{q_{B}} \xi_{i j} V_{j}
$$


where subscript $A$ is associated with directions $\theta=\frac{\pi}{4}, \frac{5}{4} \pi$, while subscript $B$ is associated with directions $\theta=\frac{3}{4} \pi, \frac{7}{4} \pi$. The measure unit of the bond constants is $\left[\frac{\mathrm{N}}{\mathrm{m}^{6}}\right]$.

In Eq. (5), $i$ and $j$ refer to a generic particle and its neighbour respectively, $V_{j}$ denotes the volume of particle $j, \xi_{i j}$ is the initial length of the bond between particles $i$ and $j$, and $q_{A}$ and $q_{B}$ represent the number of peridynamic bonds along the directions associated with $A$ and $B$, respectively.

The model was fully validated by using the FEM's deformation field as a reference, and a good agreement between the two approaches was observed (De Meo et al.). The critical stretch parameter for peridynamic bonds was obtained based on the expression given in (Madenci and Oterkus, 2014):

$$
s_{0}=\sqrt{\frac{4 \pi G_{c}}{9 E \delta}}
$$

where $E$ is the Young's modulus. In case of linear elastic material and plane stress configuration, the critical energy release rate $G_{c}$ can be obtained from the fracture toughness, $K_{I c}$, as follows (Anderson, 2005):

$$
G_{c}=\frac{K_{I c}^{2}}{E}
$$

\subsection{Peridynamic hydrogen-diffusion model}

Similar to the approach adopted in (Rimoli and Ortiz, 2010), in this study, hydrogen grain boundary diffusion is described by considering type C kinetic regime (cf. (Harrison, 1961)) using the widely accepted Fisher model (Fisher, 1951). In fact, according to Mishin and Herzig (Mishin and Herzig, 1999), the Fisher model is still the foundation of grain boundary diffusion theory. As described in (Harrison, 1961), in the case of type C grain boundary diffusion kinetic regime, the bulk diffusion of impurities is negligible with respect to the diffusion occurring along the grain boundaries. As explained in (Rimoli, 2009), this behaviour can be mathematically described by the following reduced version of the Fisher diffusion equation: 


$$
\frac{\partial C}{\partial t}=D_{g b} \Delta C
$$

where $D_{g b}$ is the grain boundary diffusion coefficient, $t$ is time, $C$ refers to the hydrogen concentration at the grain boundaries and $\Delta$ is the Laplace operator. In this study, $D_{g b}=0.84 \cdot 10^{-9} \mathrm{~m}^{2} / \mathrm{s}$ is consistent with (Rimoli and Ortiz, 2010) and (Serebrinsky et al. 2004).

As mentioned earlier, a novel peridynamic grain boundary hydrogen diffusion formulation, based on the Fisher model, is introduced. By making an analogy with the peridynamic heat conduction equation given in (Oterkus et al., 2014), the peridynamic governing equation for hydrogen diffusion can be written as:

$$
\dot{C}(\mathbf{x}, t)=\int_{H_{\mathbf{x}}} f_{\mathrm{d}}\left(C(\mathbf{x}, t), C\left(\mathbf{x}^{\prime}, t\right), \mathbf{x}^{\prime}, \mathbf{x}, t\right) \mathrm{dV}_{\mathbf{x}^{\prime}}
$$

where $\dot{C}(\mathbf{x}, t)$ is the time derivative of hydrogen concentration associated with the generic material point $\mathbf{x}$. In Eq. (9), the peridynamic function $f_{\mathrm{d}}\left(C(\mathbf{x}, t), C\left(\mathbf{x}^{\prime}, t\right), \mathbf{x}^{\prime}, \mathbf{x}, t\right)$ is called hydrogen diffusion response function and is defined as:

$$
f_{\mathrm{d}}=d \frac{C\left(\mathbf{x}^{\prime}, t\right)-C(\mathbf{x}, t)}{\left|\mathbf{x}^{\prime}-\mathbf{x}\right|}
$$

in which the peridynamic diffusion bond constant $d$ can be expressed in terms of the grain boundary diffusion coefficient as:

$$
d=\frac{6 \cdot D_{g b}}{\pi \cdot h \cdot \delta^{3}}
$$

where $h$ refers to the thickness of the plate. As mentioned earlier, the type $\mathrm{C}$ grain boundary diffusion kinetic regime means pure grain boundary diffusion, with negligible leakage and diffusion in the bulk. As a consequence, the peridynamic hydrogen diffusion model is constituted by only those bonds that cross the grain boundaries of the material.

\subsection{Hydrogen coverage boundary condition}

According to (Hirose and Mura, 1984), hydrogen atoms are generated by the corrosion at the notch root and are adsorbed by the material. The chemical and physical interactions between 
material and corrosive solution are complex and particularly challenging to model. According to (Rimoli and Ortiz, 2010), it is widely reckoned that these interactions are not entirely understood at present, and, as argued in (Scheider et al., 2008), it is not currently possible to obtain a direct measure of hydrogen concentration in the region adjacent to the crack tip. As described in (Serebrinsky et al., 2004) and in (Sofronis and McMeeking, 1989), an alternative approach, adopted in different studies, such as (Scheider et al., 2008), (Raykar et al., 2011), (Alvaro et al., 2014a) and (Olden et al., 2014), consists in assuming that hydrogen adsorption can be represented via appropriate boundary conditions. For this purpose, the concept of hydrogen coverage $\psi$ is introduced and defined as:

$$
\psi=\frac{S H C}{S H C S V}
$$

where SHC and SHCSV refer to the surface hydrogen concentration at the crack tip and the surface hydrogen concentration saturation value, respectively.

As described in Section 5, the numerical results produced by the present study are compared against the experimental results reported in (Hirose and Mura, 1984), where all the surfaces but the crack tip region of a compact tension specimen were painted before the exposure to the aqueous solution and to the action of the mechanical load. As shown in (Rimoli and Ortiz, 2010), in order to model this condition, a unit value hydrogen coverage along the crack flanks in the region adjacent to the crack tip and a zero value hydrogen coverage on all the remaining surfaces of the specimen can be applied to the model.

\section{$3.4 \quad$ Coupling of peridynamic micro-mechanical and hydrogen-diffusion model}

The coupling of diffusion field and micro-mechanical field is necessary to quantify the amount of material strength degradation due to the local presence of hydrogen and the resulting fracture behaviour. In other words, the adsorption and flow of hydrogen along the grain boundaries of the material is expected to generate a phenomenon of local embrittlement that will change the material response to the applied loading condition. In fact, as described by Lynch in (Lynch, 2011b), the hydrogen AIDEC mechanism is expected to weaken the interatomic bonds leading to local decohesion. The approach adopted in this study builds on earlier work by (Serebrinsky et al., 2004), where an impurity-dependent cohesive model informed by first-principle calculations was presented. For this purpose, DFT investigations on the decohesion of $\mathrm{BCC} F(110)$ surfaces exposed to different levels of hydrogen coverage 
carried out in (Jiang and Carter, 2004) were considered and coupled with a UBER form interplanar traction-separation relation (Rose et al., 1984) and a renormalization procedure (Serebrinsky et al., 2004) to address the lengthscale gap between the engineering and the atomistic scale associated with the first-principle calculations. The resulting impuritydependent model is described by the following equation (Serebrinsky et al., 2004):

$$
\sigma_{c}(\psi)=\sigma_{c}(0)\left(1-1.0467 \psi+0.1687 \psi^{2}\right)
$$

where $\sigma_{c}(\psi)$ represents the peak or critical stress associated with a generic level of hydrogen coverage $\psi$ and $\sigma_{c}(0)$ refers to the peak stress associated with a zero value of hydrogen coverage. As mentioned earlier, the mechanical behaviour of HSLA steel can be represented by a linear elastic model (Anderson, 2005). Moreover, considering direct proportionality between $\sigma_{c}(\psi)$ and the critical stretch $s_{0}(\psi)$, the variation of the critical stretch as a function of $\psi$ can be written in the same form of Eq. (13) as:

$$
s_{0}(\psi)=s_{0}(0)\left(1-1.0467 \psi+0.1687 \psi^{2}\right)
$$

In other words, the original critical stretch $s_{0}(0)$ decreases as the local hydrogen concentration increases. In qualitative terms only, Fig. 6 shows the constant mechanical stretch to which a generic peridynamic bond is subjected (red line) and the decrement of critical stretch with increasing hydrogen coverage (blue line). 


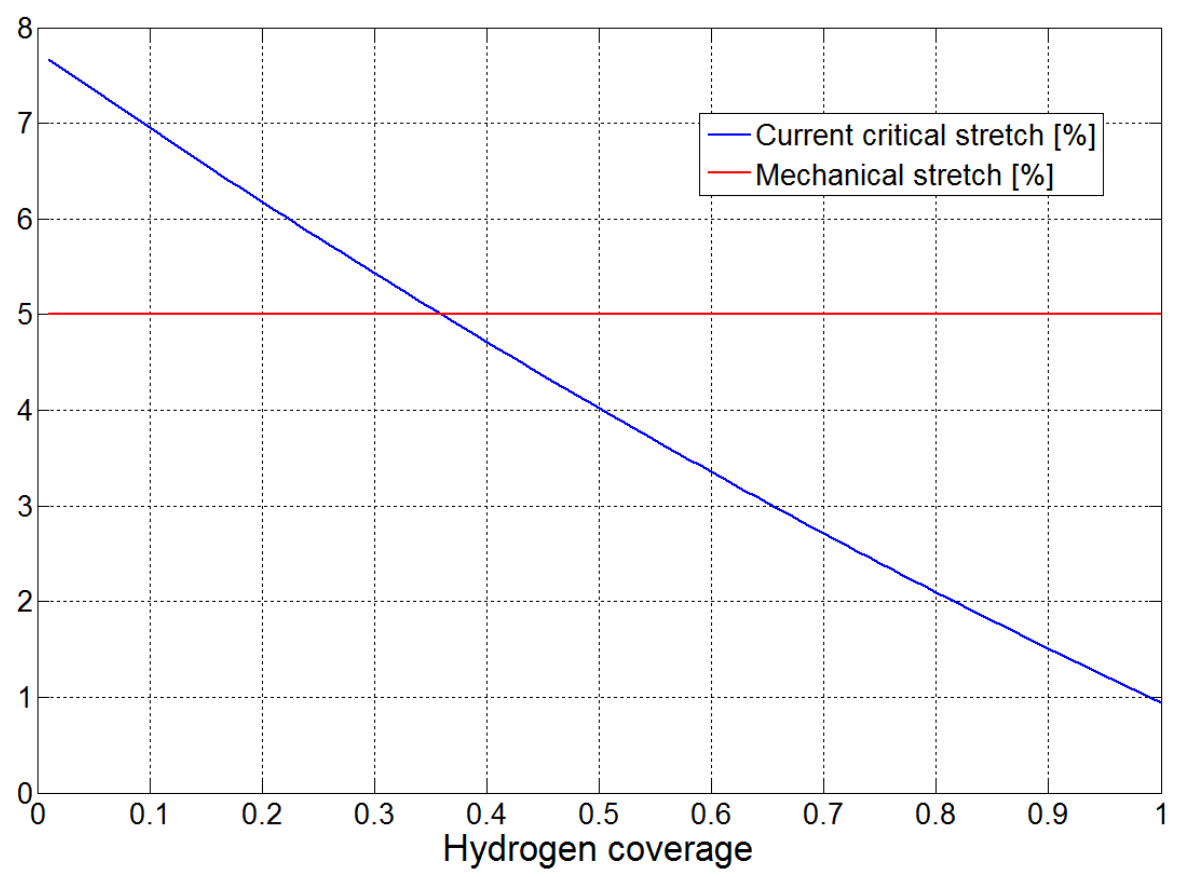

Fig. 6 - Break-even point (green circle) for the rupture of a generic peridynamic bond.

In summary, the present approach can be represented by the diagram in Fig. 7 and described by the following steps:

1) Mechanical step: a displacement-controlled load is applied at the bottom and top surfaces of the specimen until the desired stress intensity factor is reached, and it is kept constant throughout the simulation until fracture initiation.

2) Hydrogen coverage step: a unit value and a zero value hydrogen coverage are imposed at the crack tip and over all the remaining surfaces of the specimen, respectively. 3) Diffusion step: the peridynamic diffusion model is used to predict the diffusion of hydrogen along the grain boundaries of the material at each time step.

4) Embrittlement step: the relation given in Eq. (14) is used to quantify the effect of the local hydrogen concentration on the material strength. The bond under consideration is broken if its current stretch is greater than the current critical stretch.

5) Dynamic coverage step: a special algorithm is in charge of (1) locating the new position of the crack tip and (2) applying a unit value hydrogen coverage along the flanks of the newly generated crack surface. This step aims to mimic the penetration of the aqueous solution within the material as the crack advances. 


\section{Time integration}

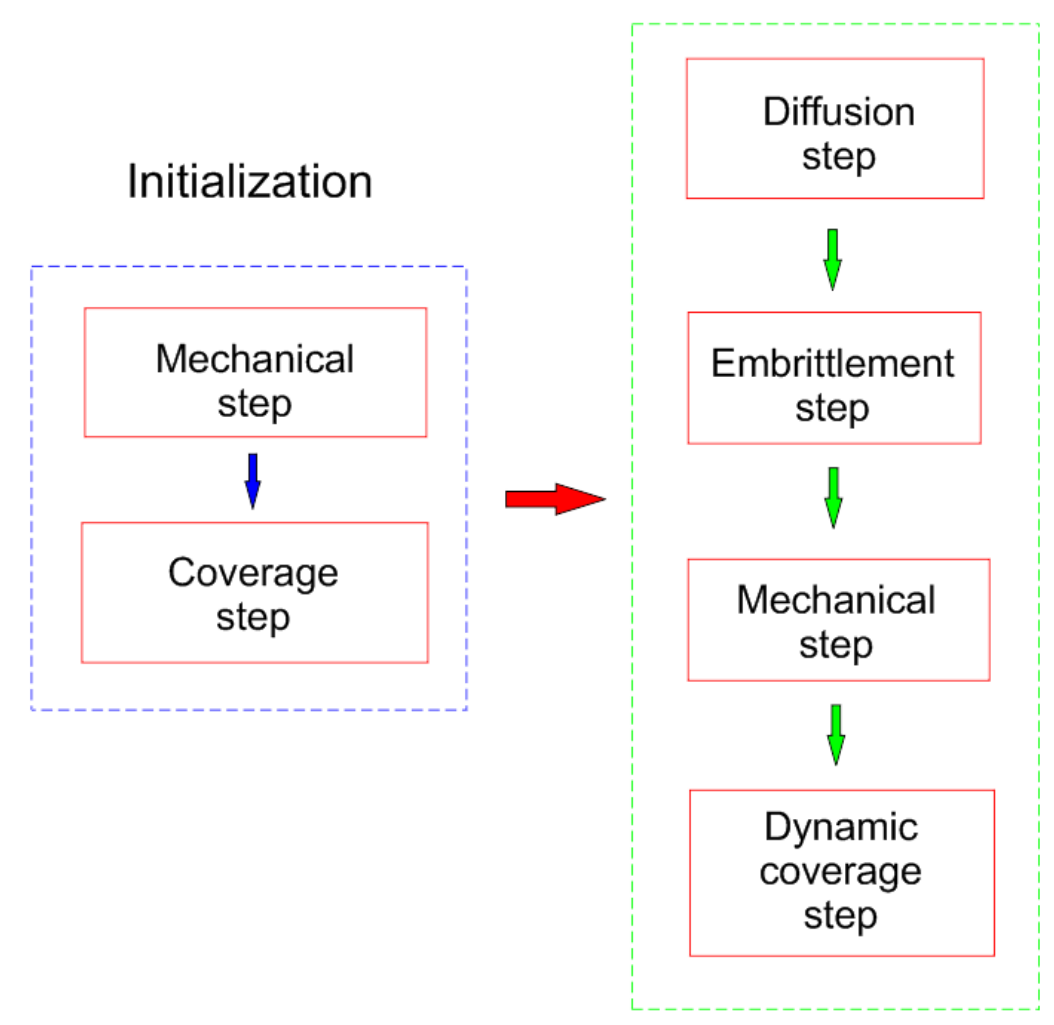

Fig. 7 - Solution procedure.

\section{$4 \quad$ Numerical results}

\subsection{Problem setup}

Similar to the case described in the experimental study (Hirose and Mura, 1984), a pre-

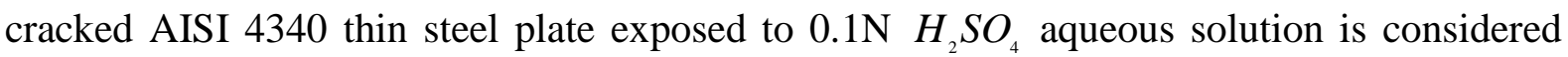
and subjected to constant displacement-controlled load applied at the bottom and top edge of the plate (Fig. 8). The load is applied statically as a boundary condition and is kept constant throughout the simulation. The plate has length of $L=2.0 \mathrm{~mm}$, width of $W=0.63 \mathrm{~mm}$ and thickness of $h=5.95 \cdot 10^{-3} \mathrm{~mm}$. The length of the initial crack is $1.25 \mathrm{~mm}$. 


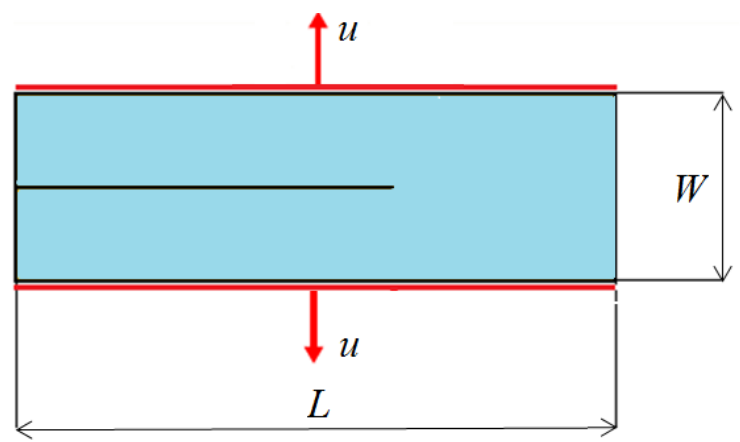

Fig. 8 - Mechanical load " $u$ ".

The magnitude of the vertical displacement-controlled load $u$ is specified as to generate a certain constant desired value of stress intensity factor at the crack tip, which is initially lower than the fracture toughness of the material (cf. Section 4.2). In other words, the initial load deforms the structure, but not at a level to generate fracture initiation. The five considered SIF values and the respective $u$ values are listed Table 1 . The displacement values were determined by performing finite element analysis and using special crack tip elements to calculate the stress intensity factors at the crack tip.

Table 1 - Loading conditions: SIF [MPa $\sqrt{\mathrm{m}}]$ vs. $u[\mu \mathrm{m}]$.

\begin{tabular}{|c|c|c|c|c|c|}
\hline SIF & 13.7 & 20.6 & 30.1 & 40.3 & 48.55 \\
\hline $\boldsymbol{u}$ & 1.157 & 1.7399 & 2.5422 & 3.4 & 4.1 \\
\hline
\end{tabular}

In addition to the vertical enforced displacement $u$, a constant unit hydrogen coverage is applied at the crack tip as described in Section 3.3.

\subsection{Material data}

The chemical composition of AISI 4340 steel considered in this study is summarized in Table 2 (Hirose and Mura, 1984):

Table 2 - Material chemical composition.

\begin{tabular}{|c|c|c|c|c|c|c|c|c|}
\hline $\mathrm{C}$ & $\mathrm{Si}$ & $\mathrm{Mn}$ & $\mathrm{P}$ & $\mathrm{S}$ & $\mathrm{Cu}$ & $\mathrm{Mo}$ & $\mathrm{Ni}$ & $\mathrm{Cr}$ \\
\hline $0.39 \%$ & $0.27 \%$ & $0.77 \%$ & $0.018 \%$ & $0.016 \%$ & $0.14 \%$ & $0.23 \%$ & $1.38 \%$ & $0.78 \%$ \\
\hline
\end{tabular}

The fracture toughness of the material is considered to be $K_{I c}=58.4 \mathrm{MPa} \sqrt{\mathrm{m}}$ (Rimoli and Ortiz, 2010). The crystals are assumed to have $\alpha$-ferrite structure with BCC lattice. As 
described in (Hosford, 1993), the local stiffness matrix of each individual crystal can be written as:

$$
[C]=\left[\begin{array}{cccccc}
C_{11} & C_{12} & C_{12} & 0 & 0 & 0 \\
C_{12} & C_{11} & C_{12} & 0 & 0 & 0 \\
C_{12} & C_{12} & C_{11} & 0 & 0 & 0 \\
0 & 0 & 0 & C_{44} & 0 & 0 \\
0 & 0 & 0 & 0 & C_{44} & 0 \\
0 & 0 & 0 & 0 & 0 & C_{44}
\end{array}\right]
$$

In order to take into account the polycrystalline nature of the material, the values of the elastic moduli $C_{i j}$ are found by applying an axial tension to a microscopic AISI 4340 steel specimen and then fitting experimental and numerical results as described in (Rimoli, 2009). The resulting microscopic material properties are:

$$
C_{11}=208.9 \quad \mathrm{GPa} \quad C_{12}=126.4 \quad \mathrm{GPa} \quad C_{44}=97.7 \quad \mathrm{GPa}
$$

\subsection{Simulation parameters}

The plate is constituted by 1,407 crystals with an approximate size of about $18 \mu \mathrm{m}$ (Fig. 4). The number of real particles along the horizontal, vertical and thickness directions is 336, 106 and 1, respectively. As suggested in (Madenci and Oterkus, 2014), three layers of virtual particles are placed along the bottom and top edges of the plate to impose the tension-loading boundary condition. The values of grid spacing and horizon's radius are $\Delta x=5.95 \mu \mathrm{m}$ and $\delta=17.94 \mu \mathrm{m}$, respectively. In order to avoid rigid body motion, the horizontal displacement of two nodes along the right edge of the plate was constrained. The time step size is $d t=1 \mathrm{~s}$ until fracture initiation. After the fracture initiation, the time step size becomes adaptive in order to keep the number of broken bonds sufficiently low in each time step which is important to allow correct redistribution of the load among the remaining bonds. For the discretization and solution of the problem, a commercial finite element software, ANSYS, is used by following the approach given in Macek and Silling (2007).

\subsection{Mechanical step}

Fig. 9 and Fig. 10 show the horizontal and vertical displacement fields, respectively, when $u=3.4 \mu \mathrm{m}$, corresponding to $\mathrm{SIF}=40.3 \mathrm{MPa} \sqrt{\mathrm{m}}$. 


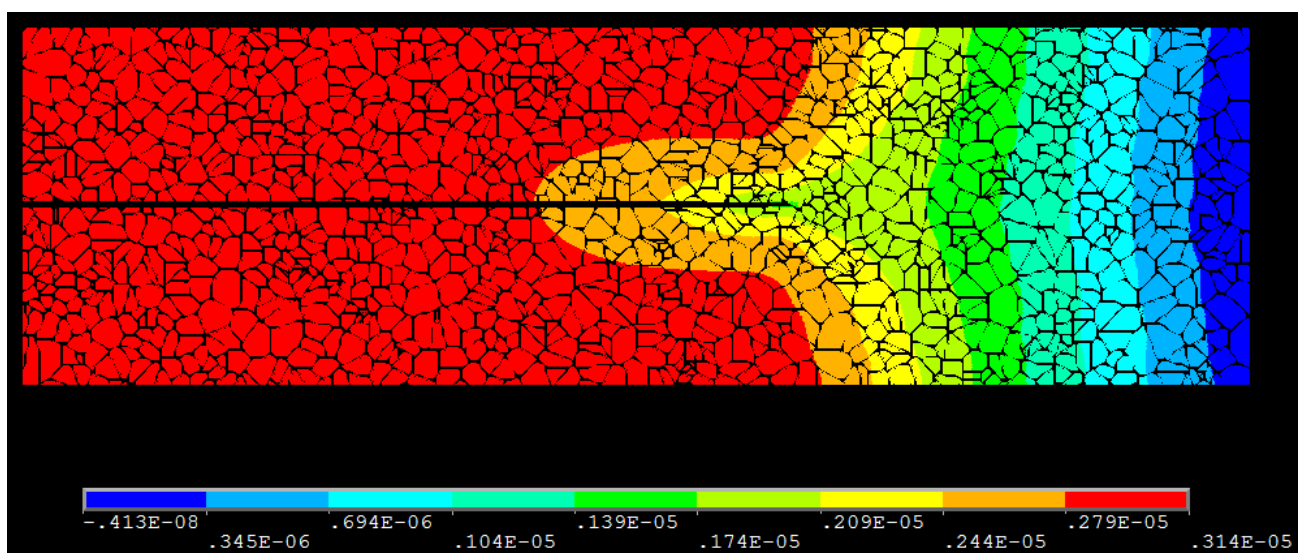

Fig. 9 - Horizontal displacement field $[\mathrm{m}]$ when SIF $=40.3 \mathrm{MPa} \sqrt{\mathrm{m}}$.

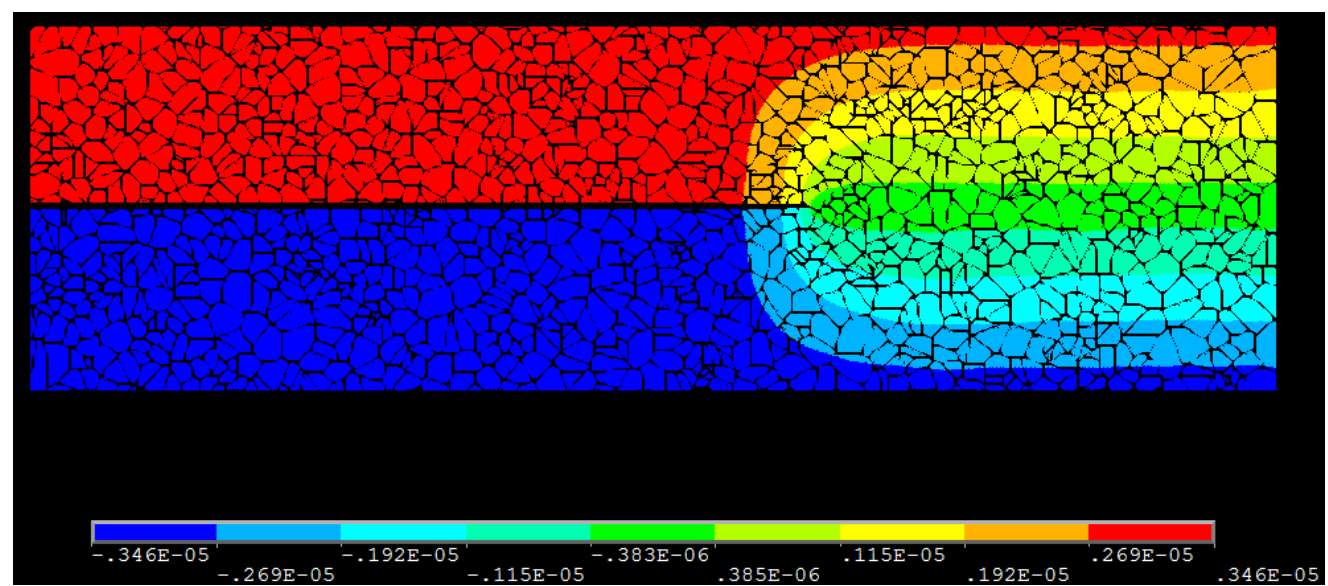

Fig. 10 - Vertical displacement field $[\mathrm{m}]$ when SIF $=40.3 \mathrm{MPa} \sqrt{\mathrm{m}}$.

As expected, due to the displacement constraints described in Section 4.3, the right hand side of the plate experiences the smallest horizontal displacements. In contrast, the opposite side of the plate shows a greater freedom of movement along the $\mathrm{x}$-axis direction. Moreover, the crack front is the region with the smallest vertical displacement, while the crack surfaces allow greater freedom of movement along the y-axis direction. As expected, the maximum stretch occurs at the crack tip (Fig. 11), and its magnitude, for all five loading conditions, is given in Fig. 12. 


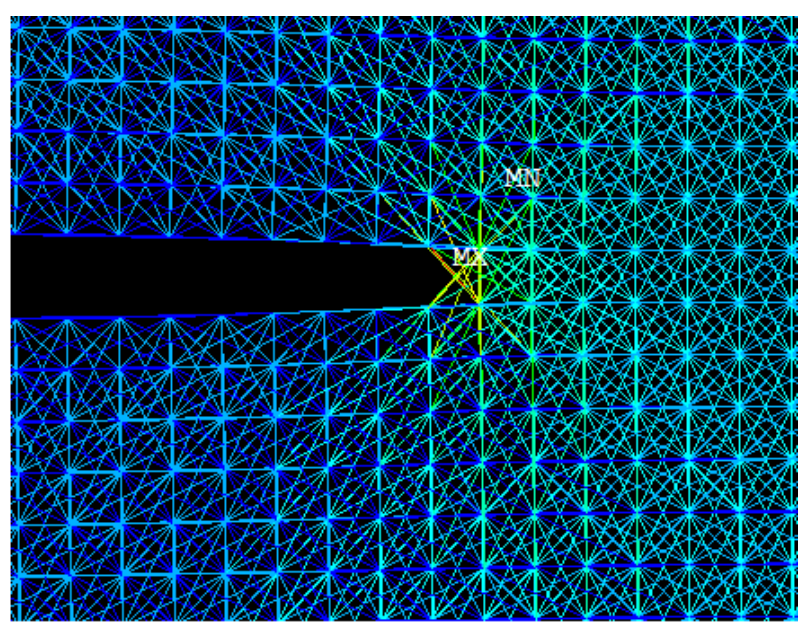

Fig. 11 - Peridynamic bonds around the crack tip and crack tip stretch when SIF $=40.3$ $\operatorname{MPa} \sqrt{\mathbf{m}}$.

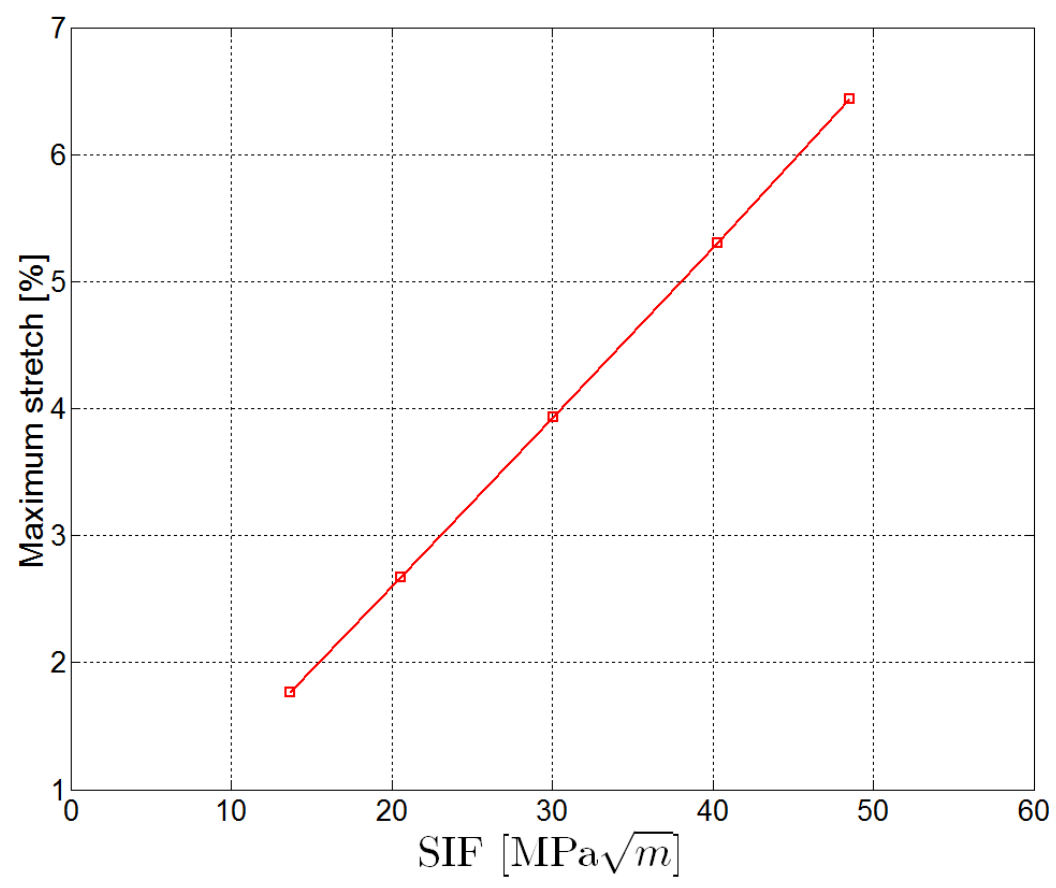

Fig. 12 - SIF $[\mathrm{MPa} \sqrt{\mathrm{m}}]$ vs. Maximum stretch [\%].

As expected, the maximum mechanical stretch increases with increasing SIF. In all five loading conditions, the mechanical stretch is inferior to the critical stretch, meaning that no crack propagation occurs at the beginning of the simulation and, as explained in Section 4.1, a reduction in critical stretch is required to obtain crack extension. 


\subsection{Hydrogen diffusion step and fracture initiation}

Fig. 13 shows the final hydrogen grain boundary coverage plots for all five loading conditions when fracture initiation occurs. The red colour refers to a unit value hydrogen coverage that corresponds to saturated hydrogen surface concentration. 

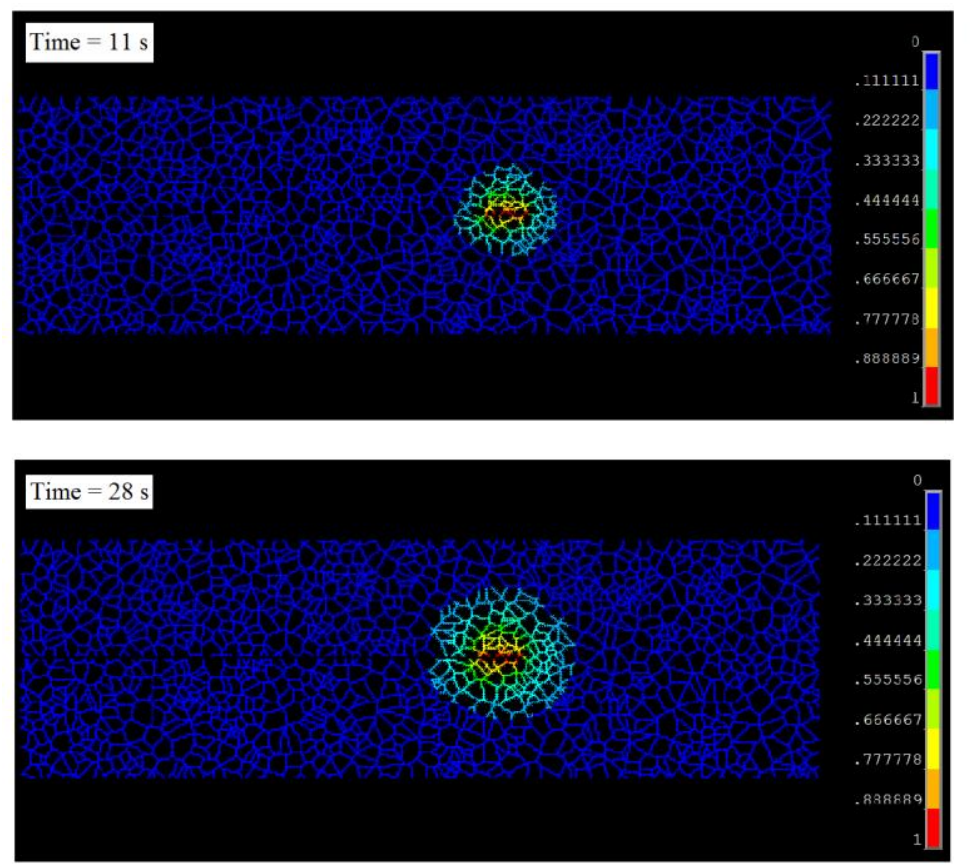

b)

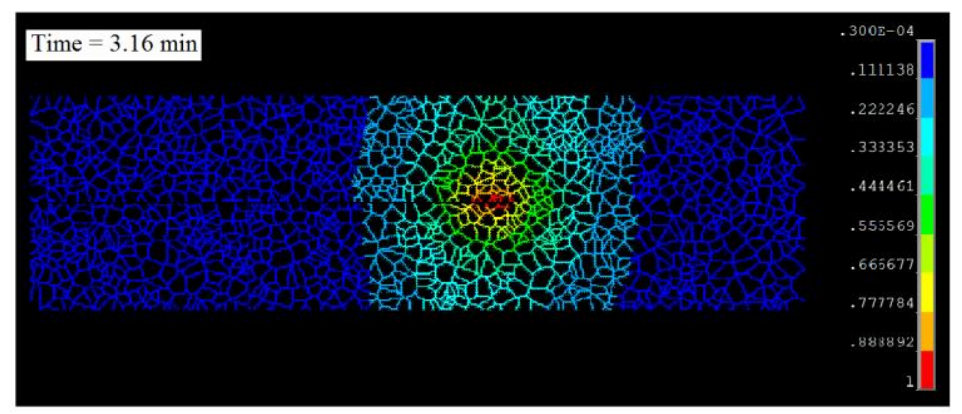

c)

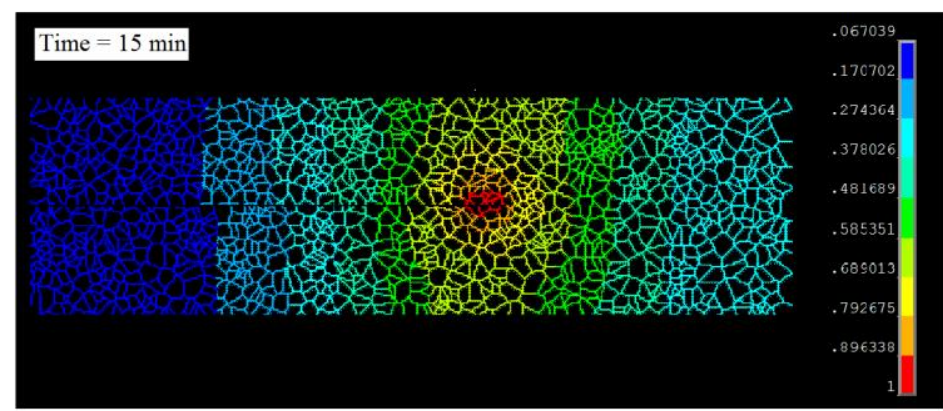

d)

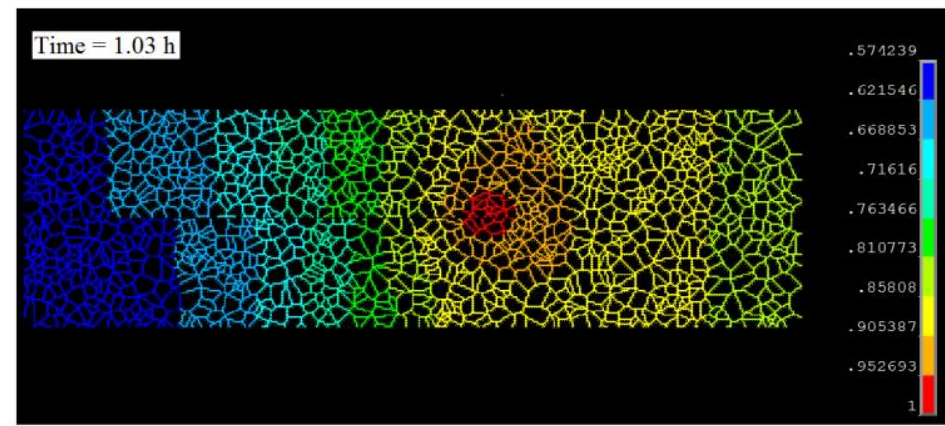

e)

Fig. 13 - Hydrogen coverage field at crack propagation initiation times for all five loading conditions: $\mathrm{SIF}=48.55 \mathrm{MPa} \sqrt{\mathrm{m}}(\mathrm{a}), \mathrm{SIF}=40.3 \mathrm{MPa} \sqrt{\mathrm{m}}(\mathrm{b}), \mathrm{SIF}=30.1 \mathrm{MPa} \sqrt{\mathrm{m}}(\mathrm{c}), \mathrm{SIF}=$ 20.6 MPa $\sqrt{\mathrm{m}}(\mathrm{d})$ and $\mathrm{SIF}=13.7 \mathrm{MPa} \sqrt{\mathrm{m}}(\mathrm{e})$. 
As time progresses, hydrogen diffuses along the grain boundaries of the material, starting from the crack tip region and yielding a local reduction in critical stretch as described in Section 3.4. As shown in Fig. 14, the higher the applied SIF, the lower the hydrogen diffusion time necessary to induce fracture initiation.

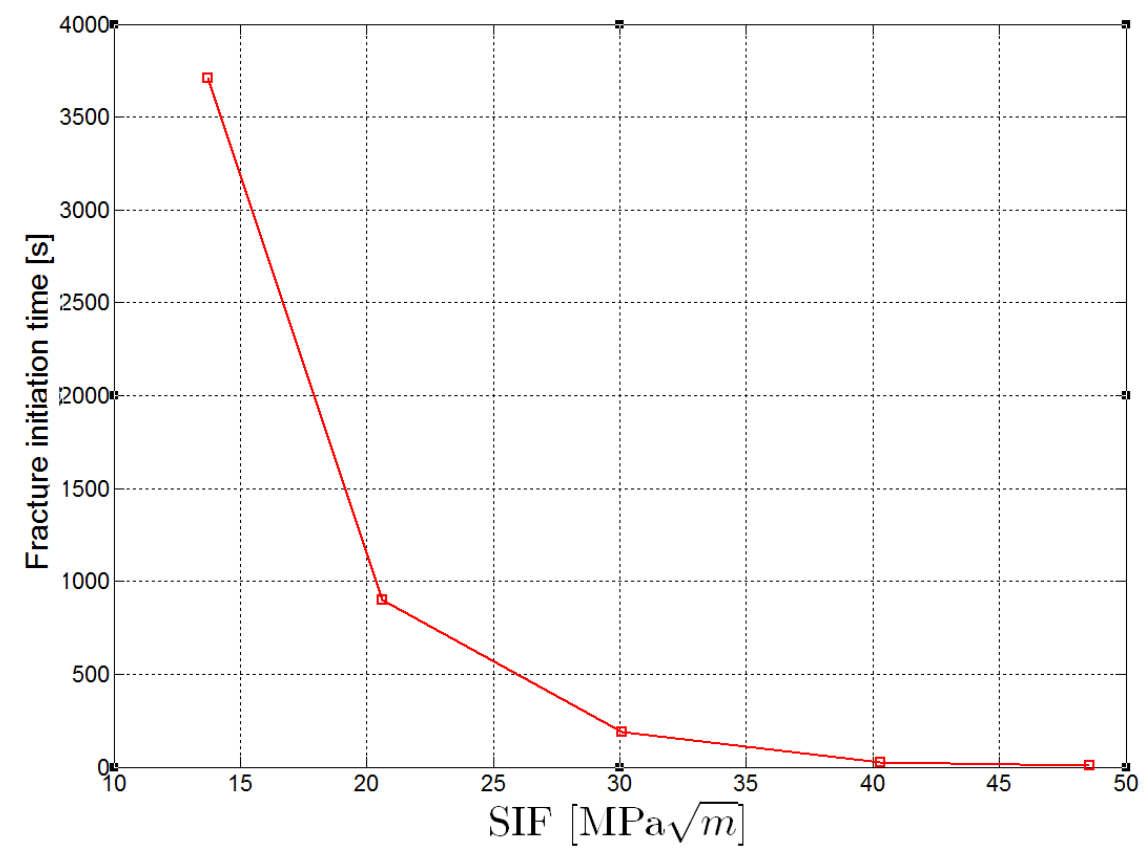

Fig. 14 - SIF $[\mathrm{MPa} \sqrt{\mathrm{m}}]$ vs. crack propagation initiation time [s].

\subsection{Crack propagation}

Fig. 15 shows the crack propagation path at three different temporal instants when SIF = 48.55 MPa $\sqrt{\mathrm{m}}$. The crack propagates from the crack tip and moves forward along the grain boundaries of the material, showing crack branching in the initial phase of the propagation. 

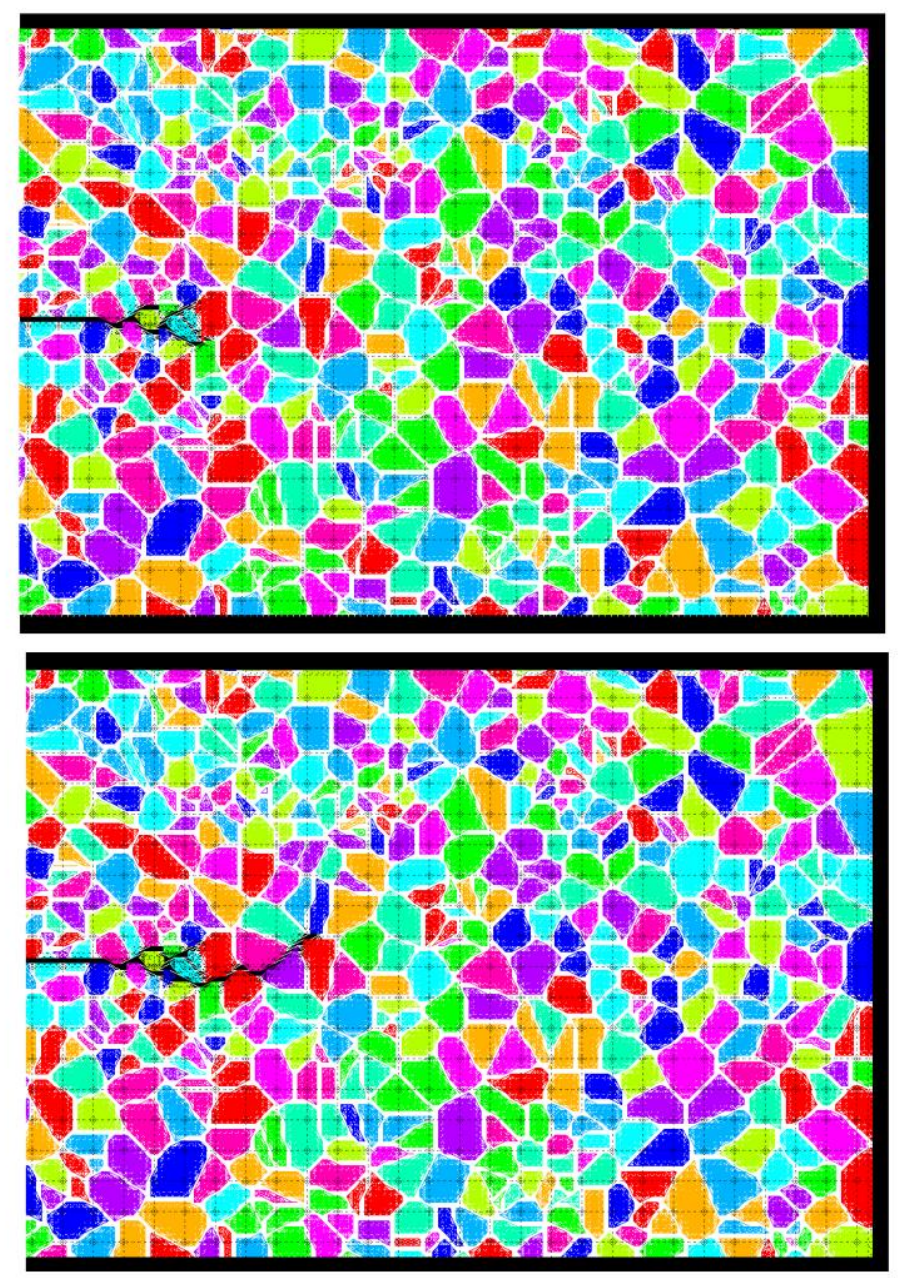

b)

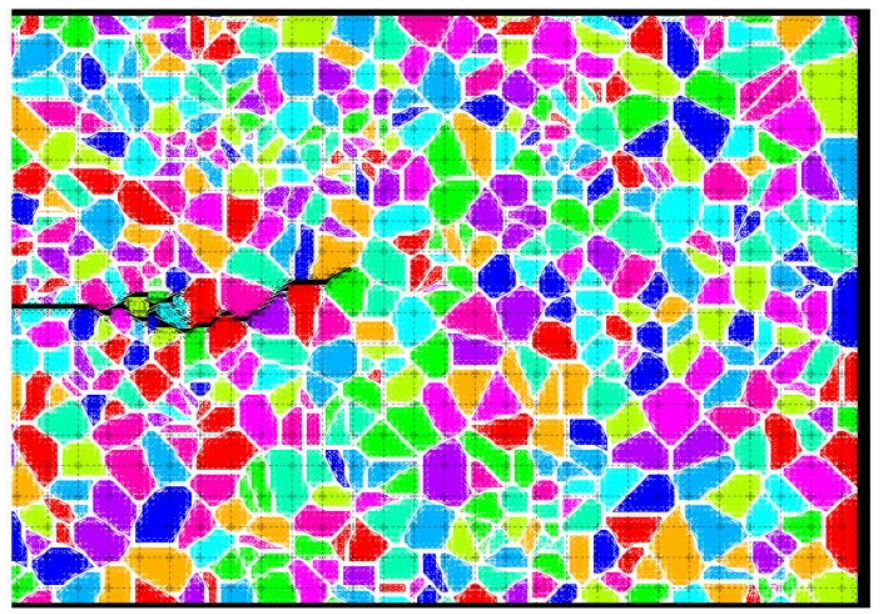

Fig. 15 - Crack propagation path when SIF $=48.55 \mathrm{MPa} \sqrt{\mathrm{m}}:$ time $=13.2 \mathrm{~s}(\mathrm{a})$, time $=16.5 \mathrm{~s}(\mathrm{~b})$, time $=18.1 \mathrm{~s}(\mathrm{c})$.

As already mentioned in Section 3.4, in order to simulate the penetration of the corrosive solution within the material as the crack advances, a dynamic hydrogen boundary condition is implemented. In other words, the constant unit value hydrogen coverage, initially applied at 
the crack tip of the initial crack, is then applied to the newly created crack surfaces as the crack extends (Fig. 16).

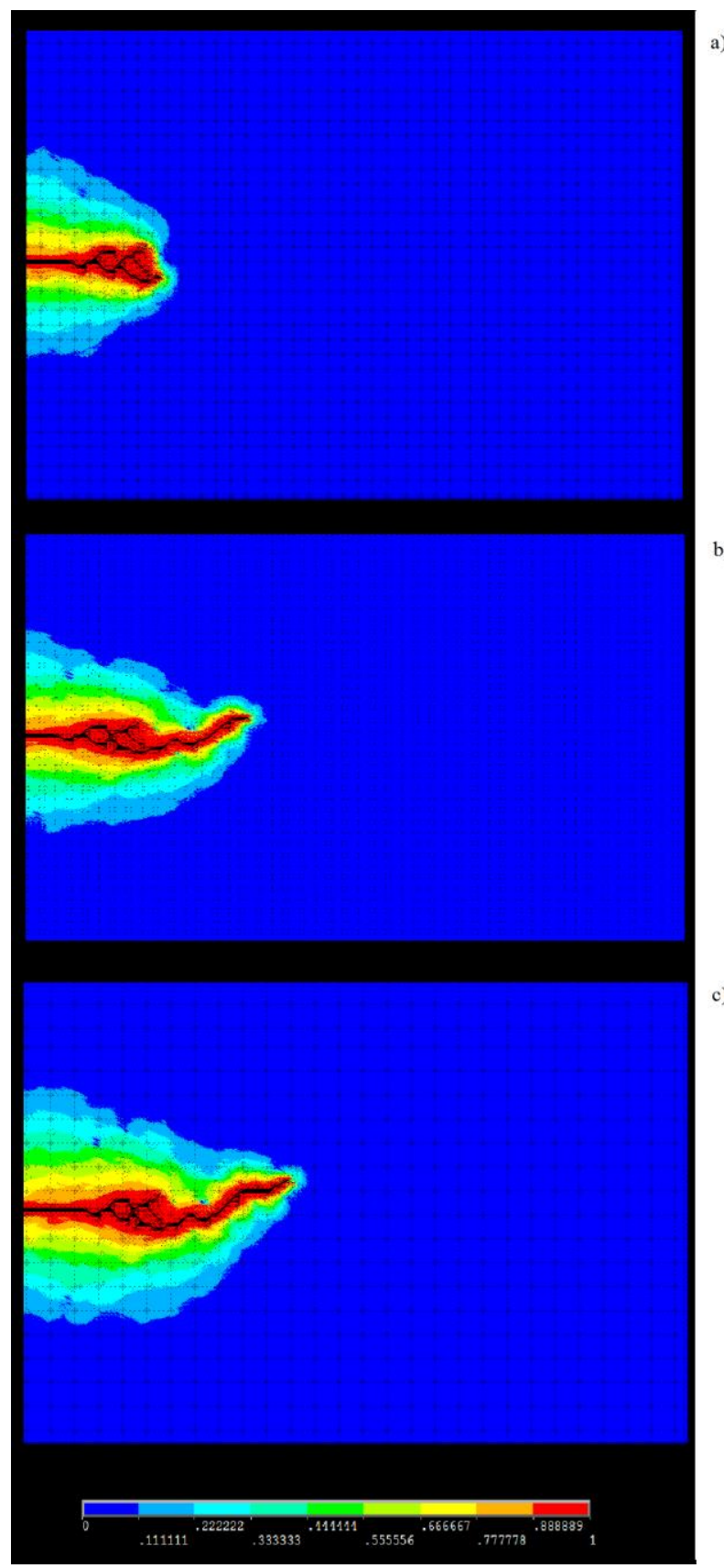

Fig. 16 - Hydrogen coverage field when SIF $=48.55 \mathrm{MPa} \sqrt{\mathrm{m}}:$ time $=13.2 \mathrm{~s}(\mathrm{a})$, time $=16.5 \mathrm{~s}$ (b), time $=18.1 \mathrm{~s}(\mathrm{c})$.

For the same loading condition, SIF $=48.55 \mathrm{MPa} \sqrt{\mathrm{m}}$, Fig. 17 shows the vertical displacement field at three different temporal instants as the crack advances. 

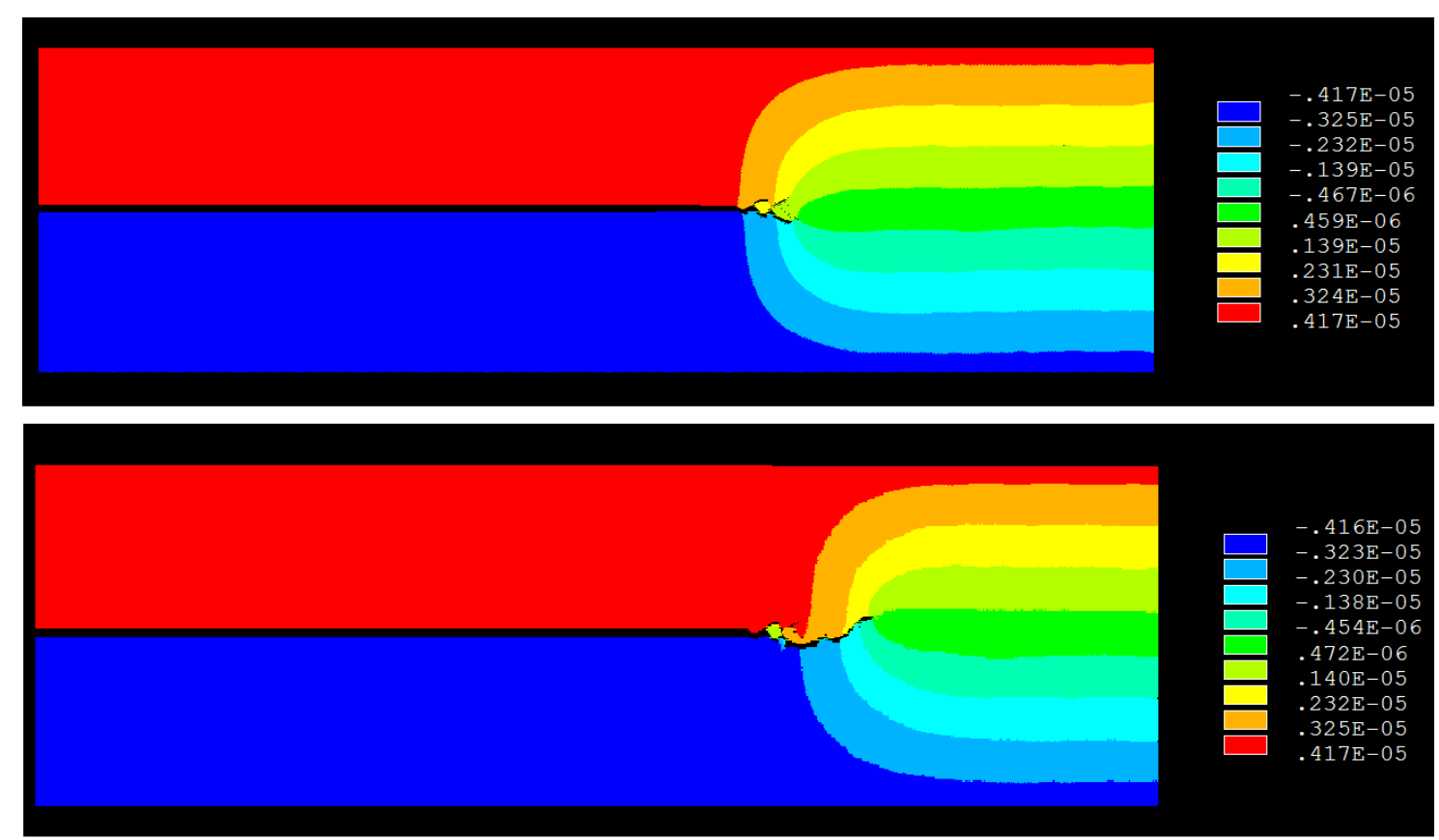

b)

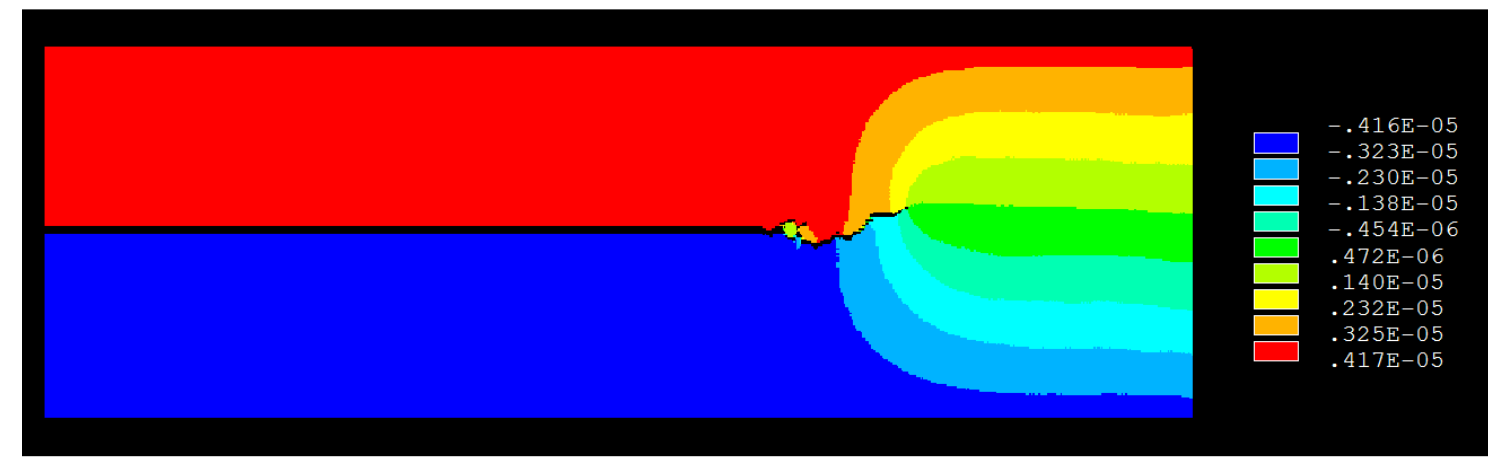

c)

Fig. 17 - Vertical displacement field when SIF $=48.55 \mathrm{MPa} \sqrt{\mathrm{m}}:$ time $=13.2 \mathrm{~s}(\mathrm{a})$, time $=16.5 \mathrm{~s}$ (b), time = 18.1 s (c).

Fig. 18 compares the crack path predicted for all five loading conditions considered in this study. A micro-branching behaviour is observed at higher values of SIF. The average micro-branching width predicted by the numerical model is on the order of $60 \mu \mathrm{m}$. 

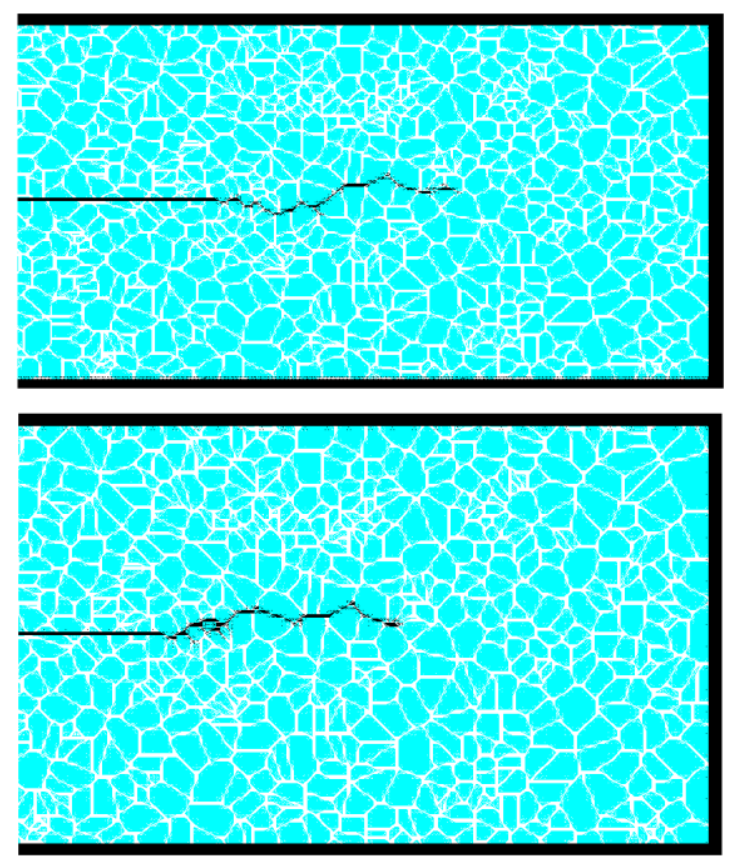

b)

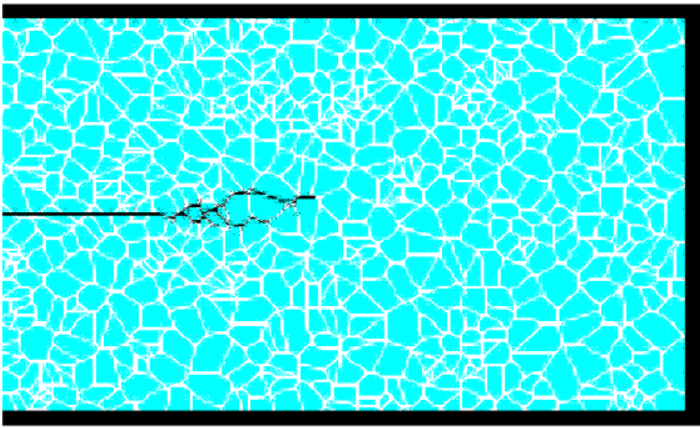

c)

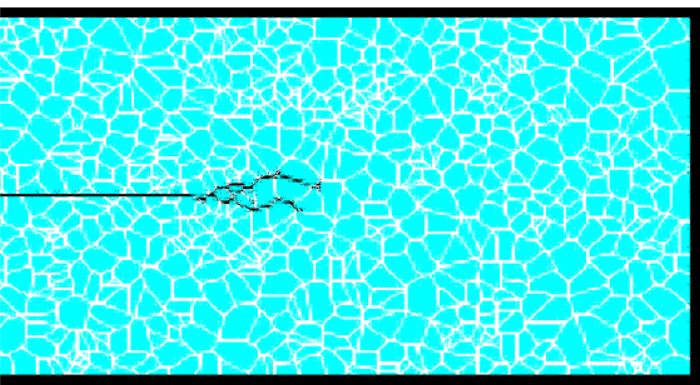

d)

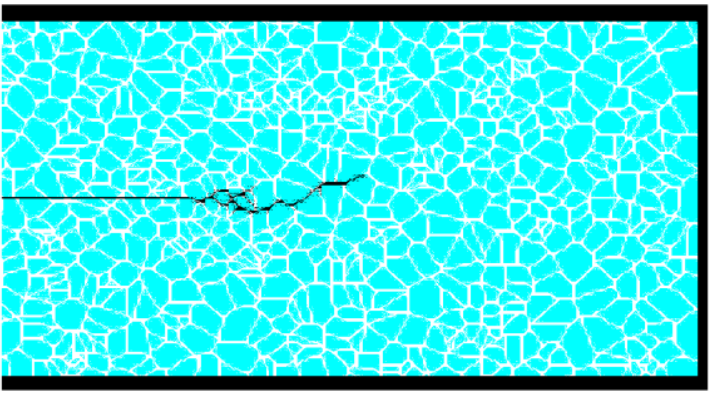

e)

Fig. 18 - Crack path: $\mathrm{SIF}=13.7 \mathrm{MPa} \sqrt{\mathrm{m}}(\mathrm{a}), \mathrm{SIF}=20.6 \mathrm{MPa} \sqrt{\mathrm{m}}(\mathrm{b}), \mathrm{SIF}=30.1 \mathrm{MPa} \sqrt{\mathrm{m}}(\mathrm{c})$, $\mathrm{SIF}=40.3 \mathrm{MPa} \sqrt{\mathrm{m}}(\mathrm{d}), \mathrm{SIF}=48.55 \mathrm{MPa} \sqrt{\mathrm{m}}(\mathrm{e})$. 
Concerning the hydrogen plot shown in Fig. 16, the algorithm is clearly able (1) to track the current position of the crack tip as the crack advances and (2) to update the hydrogen boundary condition accordingly. The vertical displacement plot shown in Fig. 17 is also in agreement with the expected behaviour of the structure: a symmetric field is found with respect to the horizontal direction, and the maximum and minimum values of the vertical displacement field are consistent with the applied mechanical load (cf. Table 1).

The validation of the current approach is made against experimental results available in ( Hirose and Mura, 1984).

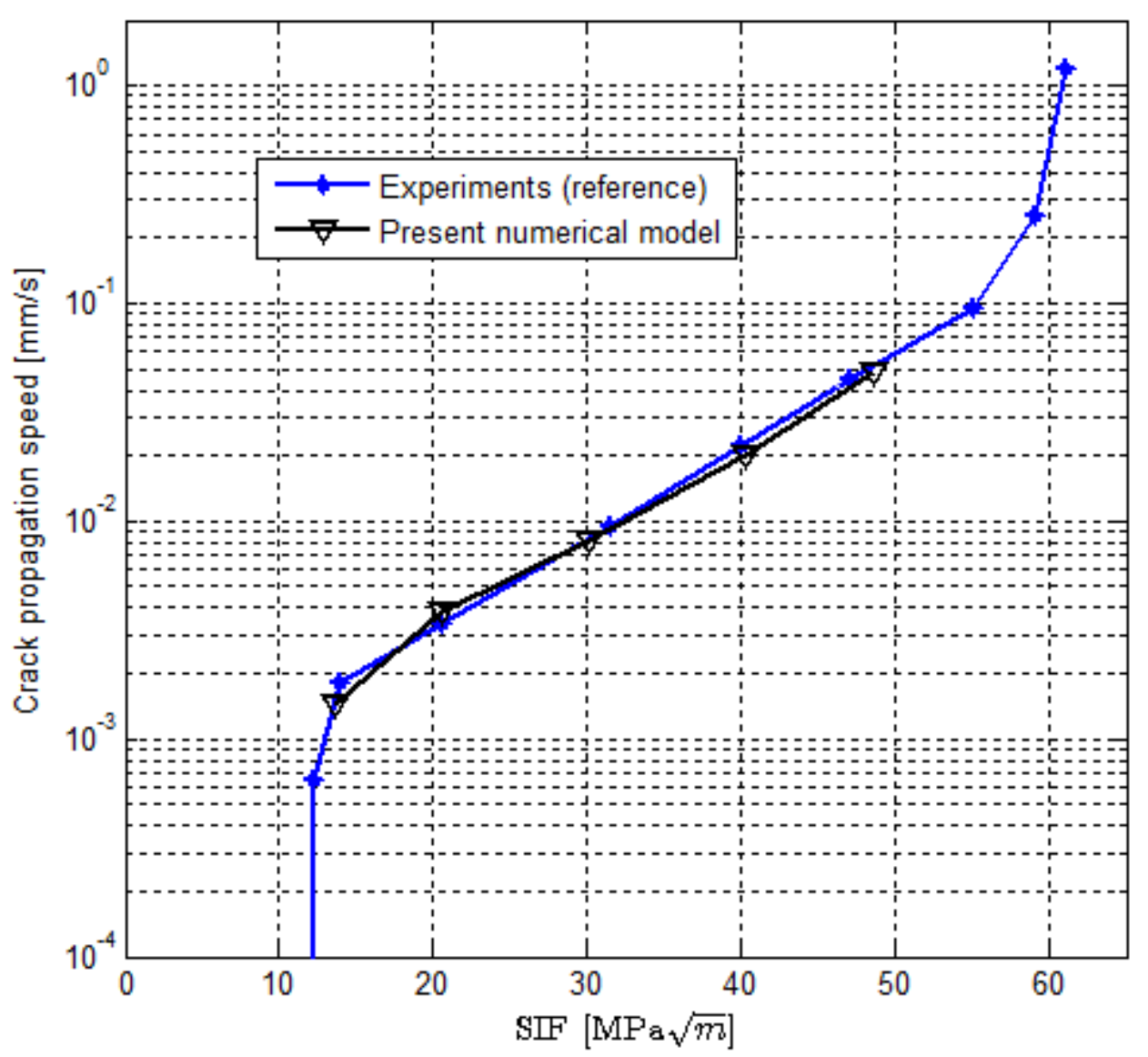

Fig. 19 - Crack propagation speed [mm/s] vs. SIF: present numerical model (black triangles) and experiments (blue diamonds).

Fig. 19 shows the comparison between the present study and experiments in (Hirose and Mura, 1984) concerning the relation between the crack propagation speed (logarithmic scale) and the stress intensity factor for all five loading conditions. The crack speed is calculated for each simulation by dividing the total crack propagation length by the crack propagation time. 
A satisfactory agreement is observed suggesting that the present peridynamic model is able to capture the main features of the complex interaction between material, environment and mechanical load.

A further agreement between numerical and experimental results is represented by the fact that an appreciable crack micro-branching behaviour is found at higher values of SIF, while for lower values of SIF the crack path is intergranular without any appreciable sign of branching behaviour (cf. Fig. 18 and (Hirose and Mura, 1984)). Moreover, the present peridynamic model is also capable of capturing the width of micro-branching observed during the experiments (cf. Section 4.6), i.e., an average $40 \mu \mathrm{m}$ of experimental microbranching width (Hirose and Mura, 1984).

\section{Conclusion}

For the first time in the literature, a numerical multiphysics peridynamic framework for modelling adsorbed-hydrogen SCC in HSLA polycrystalline steel based on the AIDEC mechanism is presented. This study extends the work of (Serebrinsky et al., 2004) and (Rimoli and Ortiz, 2010) by considering an alternative numerical technique to CZM, PD, which allows us to overcome the limitations of the former (cf. Section 1). Microstructural data are used to model the mechanical behaviour of the material and a novel peridynamic hydrogen grain boundary diffusion model is introduced.

Crack propagation speeds calculated for five different loading conditions are found in good agreement with the experimental results available in the literature. Remarkably, the peridynamic model presented here is also capable of capturing the fracture behaviour of the structure and different micro-branching behaviours and associated geometrical features (micro-branching width) observed in experiments.

Further improvements to the present study can be made by coupling the microstructural peridynamic model with methods such as electron backscatter diffraction (Pouillier et al., 2012) to measure crystal orientation with the aim to reproduce an equivalent polycrystalline model instead of producing a random texture. Moreover, a reduction in computational time can be obtained by coupling the microscopic and macroscopic mechanical model in the same peridynamic framework. Lastly, the present study can serve as a starting point for more detailed SCC investigations: more complex geometries and loading conditions, 3D models and multiple cracks. 


\section{Acknowledgement}

The authors are grateful to Prof. Siegfried Schmauder and Prof. Erdogan Madenci for the useful discussions that occurred throughout the realization of this study and acknowledge the Defence Science and Technology Laboratory (DSTL) for the financial support. A special thanks go to the anonymous reviewers, whose time and contribution have been highly appreciated. Results were obtained using the EPSRC funded ARCHIE-WeSt High Performance Computer (www.archie-west.ac.uk). EPSRC grant no. EP/K000586/1. 


\section{References}

Ahn, D.C., Sofronis, P. \& Dodds, R., 2007. Modeling of hydrogen-assisted ductile crack propagation in metals and alloys. International Journal of Fracture, 145, pp.135-157.

Alvaro, A., Olden, V. \& Akselsen, O.M., 2014a. 3D cohesive modelling of hydrogen embrittlement in the heat affected zone of an X70 pipeline steel. International Journal of Hydrogen Energy, 39(18), pp.3528-3541.

Alvaro, A., Olden, V. \& Akselsen, O.M., 2014b. 3D cohesive modelling of hydrogen embrittlement in the heat affected zone of an X70 pipeline steel - Part II. International Journal of Hydrogen Energy, 39(7), pp.3528-3541.

Anderson, T.L., 2005. Fracture Mechanics - Fundamentals and Applications 3rd ed., Boca Raton: Taylor \& Francis.

Arnoux, P., 2010. Atomistic simulations of stress corrosion cracking. Corrosion Science, 52(4), pp.1247-1257.

Askari, E. et al., 2008. Peridynamics for multiscale materials modeling. Journal of Physics: Conference Series, 125, p.012078.

Bechtle, S. et al., 2009. Grain-boundary engineering markedly reduces susceptibility to intergranular hydrogen embrittlement in metallic materials. Acta Materialia, 57(14), pp.4148-4157.

De Meo, D., Zhu, N. \& Oterkus, E., Peridynamic Modeling of Granular Fracture in Polycrystalline Materials. Journal of Engineering Materials and Technology. (Under review)

Falkenberg, R. et al., 2009. Simulation of Stress-Corrosion Cracking by the Cohesive Model. Key Engineering Materials, 417-418, pp.329-332.

Fisher, J.C., 1951. Calculation of diffusion penetration curves for surface and grain boundary diffusion. Journal of Applied Physics, 22(1951), pp.74-77.

Gerstle, W. et al., 2008. Peridynamic simulation of electromigration. Computers, Materials and Continua, 8(2), pp.75-92.

Gravouil, A., Moës, N. \& Belytschko, T., 2002. Non-planar 3D crack growth by the extended finite element and level sets-Part II: Level set update. International Journal for Numerical Methods in Engineering, 53, pp.2569-2586.

Han, G. et al., 1998. Effect of strain-induced martensite on hydrogen environment embrittlement of sensitized austenitic stainless steels at low temperatures. Acta Materialia, 46(13), pp.4559-4570.

Harrison, L.G., 1961. Influence of dislocations on diffusion kinetics in solids with particular reference to the alkali halides. Transactions of the Faraday Society, 57, p.1191. 
Hirose, Y. \& Mura, T., 1984. Growth mechanism of stress corrosion cracking in high strength steel. Engineering Fracture Mechanics, 19(6), pp.1057-1067.

Hirose, Y. \& Mura, T., 1984. Nucleation mechanism of stress corrosion cracking from notches. Engineering Fracture Mechanics, 19(2), pp.317-329.

Hosford, W.F., 1993. The mechanics of crystals and textured polycrystals, New York, USA: Oxford University Press.

Jiang, D.E. \& Carter, E.A., 2004. First principles assessment of ideal fracture energies of materials with mobile impurities: implications for hydrogen embrittlement of metals. Acta Materialia, 52(16), pp.4801-4807.

Li, Q., Zhang, Y. \& Chu, W., 2002. Molecular dynamics simulation of stress corrosion cracking in Cu3Au. Computational Materials Science, 25, pp.510-518.

Lynch, S.P., 2011. Mechanistic and fractographic aspects of stress-corrosion cracking (SCC). In V. S. Raja \& T. Shoji, eds. Stress corrosion cracking - Theory and practice. Cambridge, UK: Woodhead Publishing Limited.

Madenci, E. \& Oterkus, E., 2014. Peridynamic theory and its applications,

Macek, R. W. \& Silling, S. A., 2007. Peridynamics via finite element analysis. Finite Elements in Analysis and Design, 43 (15), pp.1169-1178.

Mine, Y. \& Kimoto, T., 2011. Hydrogen uptake in austenitic stainless steels by exposure to gaseous hydrogen and its effect on tensile deformation. Corrosion Science, 53(8), pp.2619-2629.

Mishin, Y. \& Herzig, C., 1999. Grain boundary diffusion: recent progress and future research. Materials Science and Engineering: A, 260, pp.55-71.

Moës, N., Dolbow, J. \& Belytschko, T., 1999. A finite element method for crack growth without remeshing. International Journal for Numerical Methods in Engineering, 46(February), pp.131-150.

Moës, N., Gravouil, A. \& Belytschko, T., 2002. Non-planar 3D crack growth by the extended finite element and level sets-Part I: Mechanical model. International Journal for Numerical Methods in Engineering, 53, pp.2549-2568.

Olden, V. et al., 2014. FE simulation of hydrogen diffusion in duplex stainless steel. International Journal of Hydrogen Energy, 39(2), pp.1156-1163.

Oriani, R.A. \& Josephic, P.H., 1974. Equilibrium aspects of hydrogen-induced cracking of steels. Acta Metallurgica, 22(9), pp.1065-1074.

Oterkus, E. et al., 2012. Combined finite element and peridynamic analyses for predicting failure in a stiffened composite curved panel with a central slot. Composite Structures, 94(3), pp.839-850. 
Oterkus, E. \& Madenci, E., 2012. Peridynamic Analysis of Fiber-Reinforced Composite Materials. Journal of Mechanics of Materials and Structures, 7(1).

Oterkus, S. et al., 2014. Hygro-Thermo-Mechanical Analysis and Failure Prediction in Electronic Packages by Using Peridynamics. , pp.973-982.

Pouillier, E. et al., 2012. A study of intergranular fracture in an aluminium alloy due to hydrogen embrittlement. International Journal of Plasticity, 34, pp.139-153.

Rabczuk, T. \& Belytschko, T., 2007. A three-dimensional large deformation meshfree method for arbitrary evolving cracks. Computer Methods in Applied Mechanics and Engineering, 196, pp.2777-2799.

Rabczuk, T. \& Belytschko, T., 2004. Cracking particles: a simplified meshfree method for arbitrary evolving cracks. International Journal for Numerical Methods in Engineering, 61, pp.2316-2343.

Rabczuk, T., Bordas, S. \& Zi, G., 2010. On three-dimensional modelling of crack growth using partition of unity methods. Computers \& Structures, 88(23-24), pp.1391-1411.

Rabczuk, T. \& Zi, G., 2007. A Meshfree Method based on the Local Partition of Unity for Cohesive Cracks. Computational Mechanics, 39, pp.743-760.

Raja, V.S. \& Shoji, T. eds., 2011. Stress corrosion cracking - Theory and practice, Cambridge, UK: Woodhead Publishing Limited.

Raykar, N.R., Maiti, S.K. \& Singh Raman, R.K., 2011. Modelling of mode-I stable crack growth under hydrogen assisted stress corrosion cracking. Engineering Fracture Mechanics, 78(18), pp.3153-3165.

Rimoli, J.J., 2009. A Computational Model for Intergranular Stress Corrosion Cracking.

Rimoli, J.J. \& Ortiz, M., 2010. A three-dimensional multiscale model of intergranular hydrogen-assisted cracking. Philosophical Magazine, 90(917270850), pp.2939-2963.

Rose, J.H. et al., 1984. Universal features of the equation of state of metals. Physical Review B, 29(6), pp.2963-2969.

Scheider, I., Pfuff, M. \& Dietzel, W., 2008. Simulation of hydrogen assisted stress corrosion cracking using the cohesive model. Engineering Fracture Mechanics, 75, pp.42834291.

Seleson, P. \& Parks, M., 2011. On the role of the influence function in the peridynamic theory. Internation Journal for Multiscale Computational Engineering, 9(6), pp.689706.

Serebrinsky, S., Carter, E. a. \& Ortiz, M., 2004. A quantum-mechanically informed continuum model of hydrogen embrittlement. Journal of the Mechanics and Physics of Solids, 52, pp.2403-2430. 
Silling, S. A., 2000. Reformulation of elasticity theory for discontinuities and long-range forces. Journal of the Mechanics and Physics of Solids, 48, pp.175-209.

Silling, S. A. \& Askari, E., 2005. A meshfree method based on the peridynamic model of solid mechanics. Computers and Structures, 83, pp.1526-1535.

Sofronis, P. \& McMeeking, R.M., 1989. Numerical analysis of hydrogen transport near a blunting crack tip. Journal of the Mechanics and Physics of Solids, 37, pp.317-350.

Thomas, L.E. \& Bruemmer, S.M., 2000. High-Resolution Characterization of Intergranular Attack and Stress Corrosion Cracking of Alloy 600 in High-Temperature Primary Water. Corrosion, 56(6), pp.572-587.

Traidia, A. et al., 2012. An effective finite element model for the prediction of hydrogen induced cracking in steel pipelines. International Journal of Hydrogen Energy, 37(21), pp.16214-16230.

Yagodzinskyy, Y. et al., 2010. Effect of hydrogen on plastic strain localization in single crystals of austenitic stainless steel. Scripta Materialia, 62, pp.155-158. 